\title{
Las politicas de inclusión social en Andalucía desde la perspectiva discursiva y lexicométrica. Un análisis comparativo del discurso técnico- politico
}

\author{
Social inclusion policies in Andalusia from a discursive \\ and lexicometric perspective. A comparative analysis of the \\ technical-political discourse
}

\author{
Auxiliadora González Portillo \\ Germán JaraÍz Arroyo \\ Universidad Pablo de Olavide \\ magonpor@upo.es (ESPAÑA)
}

Recibido: 20.11 .2018

Aceptado: 11.10 .2019

\section{RESUMEN}

Las políticas de inclusión social han sido analizadas desde muy diferentes perspectivas, pero en este artículo queremos presentar el análisis de las mismas desde el marco del Análisis del Discurso y la Lexicometría. Para ello nos hemos centrado en el discurso de dos de sus principales actores, aquellos que las diseñan (políticos) y aquellos que las ejecutan (técnicos), recogidos mediante entrevistas en profundidad y grupos focales que se hicieron en el trabajo de campo de un proyecto de investigación más amplio sobre la Políticas Sociales autonómicas en financiado por el por el Ministerio de Economía y Empresa (MIMECO) de España, en la convocatoria de proyectos de Investigación+Desarrollo 2014-2017.

Para el análisis del discurso desde parámetros léxicos hemos utilizado el software Iramuteq, el cual, mediante un sistema de codificación y de análisis multidimensional estadístico, nos permite profundizar y categorizar los mundos léxicos presentes en los discursos. En este caso, establecemos una comparativa entre los mundos léxicos de los políticos y de los técnicos con respecto a las políticas de inclusión social en Andalucía.

El resultado de la aplicación de esta metodología pone de manifiesto, en un primer momento, la oportunidad que ofrece la lexicometría como una primera forma de acercamiento a las representaciones sociales de los distintos actores, 
que posteriormente habrán de ser analizados en mayor profundidad con otras metodologías cualitativas. En un segundo momento, ya centrado en el caso analizado, la investigación nos muestra la presencia de dos discursos sobre las políticas de inclusión social en función del perfil del actor que lo enuncia, comprobándose en muchos casos, la distancia entre la percepción teórica (políticos) y la percepción pragmática (técnicos), así como la necesidad de que ambos sean compartidos en aras de una mejora de la eficacia de las políticas de inclusión social.

\title{
PALABRAS CLAVE
}

Análisis del discurso, Lexicometría, Inclusión Social, Políticas Sociales, Iramuteq, Representaciones Sociales.

\begin{abstract}
Social inclusion policies have been analyzed from very different perspectives, but in this article we want to present their analysis from the framework of Discourse Analysis and Lexicometry. To this end, we have focused on the discourse of two of its main actors, those who design them (politicians) and those who execute them (technicians), collected through in-depth interviews and focus groups that were done in the field work of a broader research project on autonomous Social Policies funded by MIMECO in the call for R\&D projects (2014-2017).

For the analysis of discourse from lexical parameters we have used the Iramuteq software, which, through a system of coding and statistical multidimensional analysis, allows us to deepen and categorize the lexical worlds present in the discourse. In this case, we establish a comparison between the lexical worlds of politicians and technicians with respect to social inclusion policies in Andalusia.

The result of the application of this methodology shows, at first, the opportunity offered by lexicometry as a first way of approaching the social representations of the different actors, which will later have to be analysed in greater depth with other qualitative methodologies. In a second moment, already centred on the case analysed, the research shows us the presence of two discourses on social inclusion policies according to the profile of the actor who enunciates them, proving in many cases the distance between the theoretical (political) perception and the pragmatic (technical) perception, as well as the need for both to be shared in order to improve the effectiveness of social inclusion policies.
\end{abstract}




\section{KEY WORDS}

Discourse analysis, Lexicometry, Social Inclusion, Social Policies, Iramuteq, Social Representations.

\section{INTRODUCCIÓN Y MARCO CONCEPTUAL}

El lenguaje es constructor de la realidad, por tanto, atender a su análisis, facilita las claves para entenderla desde la perspectiva del que emite ese lenguaje. Autores como Spink (2000) lo definen como una práctica social y mediadora de la construcción de la realidad, completando así la concepción de Berger y Luckmann (1985) como sistema de señales de vocales que expresan directa y claramente la subjetividad humana.

Junto a esta concepción del "lenguaje", se recupera la idea de "representaciones sociales"1 siguiendo la definición de Moscovici (1961) (citado por Segovia, Basulto y Zambrano, 2018: 82) "sistemas cognitivos con una lógica y lenguajes propios (...) No representan simples opiniones, imágenes o actitudes en relación a algún objeto, sino teorías y áreas de conocimientos para el descubrimiento y organización de la realidad". De esta forma, lenguaje y representaciones sociales van de la mano, y ha sido este binomio el que ha constituido el eje articulador de la propuesta metodológica de investigación que se presenta a lo largo de este artículo.

Una de las posibles formas de abordaje del binomio lenguaje-representaciones sociales es el análisis de las prácticas discursivas. Son varios los trabajos que han abordado la utilidad del análisis de contenido de los discursos de los actores, utilizándola como estrategia de investigación aplicándola a diversos casos o temáticas (Piñuel, 2002; Krippendorf, 2004; Kohlbacher, 2006; Kaefer et al., 2015; Lima y Manini, 2016; Stamann et al. 2016; Morales, 2016; Mendieta y Esparcia, 2017) pero, en pocas ocasiones, se ha aplicado al campo de las políticas sociales.

En general, las políticas de inclusión social han sido analizadas desde muy diversos enfoques y paradigmas, pero casi todos ellos centrados en la evaluación y los resultados de las mismas (su impacto) o bien en la normativa sobre la que se sustenta. En esta ocasión, se quiere mostrar la oportunidad de analizar las mismas desde otro enfoque, el de los discursos que sobre ellas se dan, incorporando una perspectiva multiactor. Para esta investigación se parte de la hipótesis de que las políticas llevan aparejada una gran carga de representación social que, en cierta forma, marcan el devenir de las mismas, y que se manifiesta a través de los

1 Es importante aclarar que para el desarrollo de este trabajo se trabaja con la noción de "representación social" diferenciándola del concepto de "imaginario social", es decir, si se sigue el esquema de Segovia, Basulto y Zambrano (2018: 84), en nuestro análisis nos centramos en el plano de significaciones sociales más superficial, , sin entrar en un ejercicio reflexivo-hermenéutico más profundo de las bases de la sociedad que es lo que aludiría al concepto de imaginario social (Castoriadis, 1989). 
discursos de los distintos actores que participan en ella. En este sentido, el objetivo de esta investigación es identificar desde una perspectiva comparada, cuales son los discursos del staff político y del staff técnico en relación a las políticas sociales en Andalucía. Para ello se llevará cabo una metodología mixta en la que, basándose en un primer momento en un análisis cuantitativo desde la perspectiva de la Lexicometría (mediante el software Iramuteq de análisis estadístico), se pasará en un segundo momento a un análisis cualitativo-interpretativo sobre los discursos de representación, que tendrá en cuenta, además del léxico, la dimensión contextual relacional donde el mismo se produce.

Profundizando en esta propuesta de analizar las políticas desde otra perspectiva, autores como Zittoun (2009) defiende que el análisis de políticas públicas debería enfocarse en algo más que el aspecto objetivo del cambio, como por ejemplo los aspectos subjetivos o discursivos. En la misma línea, Taina Saarinen (2005) señala que el análisis de discurso en el ámbito de la política pública es adecuado, en la medida en que no presenta apenas descripciones, sino que construyen también explicaciones, entendimientos y, por ende, significados. Al deconstruir el discurso político, en aras de identificar relatos explícitos e implícitos, permite comprender el significado de las mismas palabras, o similares, cuando se utilizan en diferentes contextos (Lima y Rubaii, 2016), que es lo que se pretende mostrar en este artículo mediante la comparativa de los contextos de los diferentes actores que entran en juego en las políticas de inclusión social.

En este sentido, los discursos que sobre estas políticas están más presentes en los imaginarios colectivos provienen de dos fuentes principalmente, por un lado, aquellos que provienen del sector político, en la medida que son ellos los que diseñan estas políticas y los que tiene más presencia en los medios de comunicación y, por otro lado, el discurso que proviene de los propios perceptores de esas políticas, es decir, los ciudadanos, cuyo discurso los tenemos presentes en los espacios de la cotidianidad y, hoy en día, a través de las redes sociales. Pero existe un discurso de representación que está muy invisibilizado a nivel de imaginario colectivo, y es el que proviene de los propios técnicos, de los profesionales que ejecutan esas políticas, ocupando una posición bisagra entre los que las diseñan (políticos) y los que las reciben (ciudadanos).

El análisis de los llamados "discursos profesionales" es un área de creciente interés en los estudios actuales del discurso (Bazerman \&Paradis, 1991; Bathia, 1993; Gunnarson, 2000; Trosborg, 2000). Como argumenta López Ferrero (2002), en una sociedad cada vez más especializada, tecnificada, en donde se exige a los profesionales un eficaz dominio de los discursos que genera su actividad, es necesaria una atención específica a estas formas de comunicación, discursos variados y en constante evolución, sensibles a los cambios sociales y culturales, y es por ello que este discurso profesional se ha recuperado ocupando un lugar preeminente en esta investigación.

A la hora de profundizar en el estudio del análisis de las prácticas discursivas, a nivel teórico se abordan tres grandes corrientes. Por un lado, la corriente de Análisis del Contenido liderada por Bardin (1996) (aunque fue Berelson (1952) el primero en referirse al análisis de contenido como técnica de investiga- 
ción), por otro lado, la corriente de Análisis del Discurso de origen francés, liderada por Maingueneau (2014) y Paltridge (2012), y por último la Aproximación Lingüístico-textual tradicional, entre los que se encuentra Lérat (1995).

En la primera de las corrientes, Berelson (1952) definió el Análisis de Contenido como una técnica de investigación para la descripción objetiva, sistemática y cuantitativa del contenido de la comunicación, aunque pudiera ser utilizada en otros campos como en el análisis cualitativo de variables o en el estudio de la complejidad de un fenómeno, entre otros. En la misma línea, y con posterioridad Bardin (1996) lo define como el "conjunto de técnicas de análisis de las comunicaciones, tendentes a obtener indicadores (cuantitativos o no) por procedimientos sistemáticos y objetivos de descripción del contenido de los mensajes, permitiendo la inferencia de conocimientos relativos a las condiciones de producción /recepción (contexto social) de estos mensajes" (Bardin 1996:32). Bardin (1996) incorpora a la definición de Berelson (1952) la importancia del análisis de las condiciones del contexto de producción y recepción, aspecto éste que resulta de suma importancia en el caso investigado que aquí se presenta, de ahí que esta aproximación teórica se apoye más en las aportaciones de Bardin (1996). En este caso de estudio el procedimiento sistemático nos lo ofrece el software utilizado en el análisis de los corpus textuales, Iramuteq, basado en las aportaciones de Reinert $(1987 ; 1998 ; 2003)$ que defiende que el análisis estadístico de los discursos tiene pleno sentido ya que permite localizar repeticiones frecuentes de léxicos que tienen una organización habitual, para referirse a un mismo objeto. Con respecto a las inferencias interpretativas a partir de las condiciones de producción del texto, éstas vienen determinadas por la variable político /técnico, es decir por las condiciones diferentes que se entiende que se dan entre los que diseñan las políticas de inclusión social (*perfil_pol) y los que las ejecutan (*perfil_tec).

La corriente francesa, también refuerza esta idea anteriormente plasmada ya que, para Maingueneau (2014), el Análisis del Discurso consiste en remitir los discursos a los lugares sociales /institucionales donde se produjeron, es decir, el espacio político o el espacio técnico. En la misma línea Paltridge (2012) sostiene que el Análisis del Discurso va más allá de la descripción de los usos lingüísticos a nivel microtextual y contribuye, no solo a describir la selección léxica del hablante, sino también a explicarla.

Por otro lado, la aproximación Linguístico-Textual Tradicional (Lérat, 1995) se centra en la descripción de los rasgos lingüísticos, abordando los discursos desde los diferentes niveles lingüísticos: léxico, morfológico y sintáctico. Para esta investigación, se toma de esta corriente lo relativo al análisis del léxico (López, 2002), centrándose en el análisis del uso de una determinada terminología según las variables (políticos / técnicos) así como las combinaciones de palabras o colocaciones que, por su recurrencia en determinados discursos, terminan caracterizando la forma de expresión de estos ámbitos (políticos y técnicos).

Además de la influencia de estas tres corrientes, se puede abordar el análisis de las prácticas discursivas desde los aportes de Palmquist (2010) que establece dos grandes tipos o métodos complementarios entre sí, el análisis conceptual y el 
análisis relacional. El primero de ellos se corresponde con el planteamiento más generalizado del análisis de contenidos y se centra en la detección de conceptos o términos explícitos e implícitos en un determinado documento, y su posterior análisis (con frecuencia cuantitativo) en cuanto a la presencia e importancia que cada concepto tiene en ese documento. Por su parte, el análisis relacional o semántico se inicia, al igual que el análisis conceptual, con la identificación de conceptos presentes en un documento; pero a diferencia del anterior, éste se centra en las relaciones entre los conceptos o significados de los conceptos. A lo largo de este artículo y de la metodología desarrollada se lleva a cabo la implementación de ambos métodos.

Por último, en este marco conceptual introductorio es importante aclarar que el trabajo que aquí se presente forma parte de un proyecto más amplio, el proyecto "Políticas de inclusión en la Comunidades Autónomas. Ubicación en el contexto europeo y respuesta a las nuevas situaciones" (CSO2014-51901 -P), financiado por el Ministerio de Economía y Empresa (MIMECO) de España, en la convocatoria de proyectos de Investigación+Desarrollo 2014-2017. El objetivo del mismo ha sido analizar las reformas en las políticas autonómicas de inclusión orientadas a los sectores excluidos (rentas mínimas y políticas de activación) y sus efectos, estudiando cómo se enfrentan al nuevo escenario acaecido a raíz de la crisis económica, laboral y social que se desencadenó en España en 2008 y que perfiló un escenario de grandes transformaciones. Igualmente se ha pretendido identificar experiencias innovadoras con el objetivo de construir políticas de inclusión más eficientes y adaptadas a las nuevas necesidades. Para conseguir este objetivo se ha llevado a cabo un trabajo de campo basado en una metodología cualitativa (grupos focales y entrevistas en profundidad) a diferentes actores (políticos, técnicos y población perceptora) de las diferentes Comunidades Autónomas. Para este caso, el material en el que se basa este artículo es el procedente del trabajo de campo hecho en la Comunidad Autónoma de Andalucía.

Este proyecto ha dado lugar a una serie de resultados vinculados a la evaluación de estas políticas, sus fortalezas y sus retos que han sido ya extraídos y está en proceso de publicación en diferentes formatos. Pero dado que no se dispone de muchos trabajos que apliquen el análisis del discurso a las políticas de inclusión social, el objetivo de este artículo es mostrar e ilustrar la oportunidad de la aplicabilidad de este marco metodológico a este campo y, por tanto, se centrará en los aportes metodológicos y no tanto en los resultados de la evaluación de las políticas de inclusión social en sí mismas.

\section{METODOLOGÍA}

A lo largo de este trabajo se presenta una propuesta de metodología mixta en la que basándose, en un primer momento, en un análisis cuantitativo desde la perspectiva de la Lexicometría se pasará, en un segundo momento, a un análisis cualitativo-interpretativo sobre los discursos de representación, que tendrá en cuenta, además del léxico, la dimensión contextual relacional donde el mismo 
se produce. Entendemos que ambas aportaciones resultan sumamente complementarias y de gran interés, para el tipo de contexto que estamos analizando (los discursos sobre las políticas sociales).

En la base de la propuesta metodológica que aquí se presenta está lo que Pêcheux (1969) definía como la "materialidad discursiva", es decir, los datos textuales que se pueden identificar y observar (palabras, sintagmas nominales, nominalizaciones, expresiones idiomáticas y verbos). En la misma línea, Reinert $(1987 ; 1998 ; 2003)$ habla de los "mundos lexicales" entendiéndolos como la concatenación de palabras que componen un discurso determinado (sustantivos, adjetivos y verbos) evocados por los enunciadores, En definitiva, se trata de la Lexicometría como eje metodológico de la investigación, la cual cuenta con una larga tradición y apoyo teórico. Esta se define como la "disciplina que permite describir cuantitativamente los textos constitutivos de un corpus a partir de su segmentación en formas gráficas. Consta de un conjunto de métodos que permiten hacer reorganizaciones formales de la secuencia textual y análisis estadísticos de vocabulario" (Salem, Cabré y Romeu, 1990:26).

De esta forma, en un primer momento, se pondrá el énfasis en las unidades léxicas y su relación con los diferentes contextos multiactores (políticos/técnico) para, en un segundo momento, crear una interpretación que tenga en cuenta estos aspectos lingüísticos y sociales, y es ahí donde aparece la noción de "representación social" (Segovia, Basulto y Zambrano, 2018) al que se hacía alusión en el apartado de introducción y marco conceptual. En cierta forma, se da respuesta a la propuesta de Palmquist (2010) y sus métodos complementarios entre sí, el análisis conceptual y el análisis relacional.

Por último, resaltar que el trabajo de campo basado en entrevistas en profundidad y grupos focales son de especial importancia ya que juegan un papel central para la obtención de los discursos y por tanto del léxico que lo compone.

\subsection{Descripción del trabajo de campo.}

La obtención de los discursos base para el posterior análisis ha sido fruto de un trabajo de campo que se desarrolló entre los meses de diciembre de 2016 y marzo de 2017 en distintas provincias de Andalucía.

Conforme a los objetivos del proyecto, anteriormente descrito en el apartado introductorio, la finalidad de este trabajo de campo era aproximarse a los diferentes puntos de vista sobre las políticas de inclusión social llevadas a cabo en Andalucía, para ello resultaba esencial una selección equilibrada de entrevistados que aportaran modos diversos de enfocar la arena de estudio (Rubin \& Rubin, 1995). Se atendió a los diferentes actores que, de una u otra forma, participan en estas políticas de inclusión, ya sea diseñándolas (políticos), ejecutándolas (profesionales) o siendo receptores de las mismas (perceptores). Se ha de aclarar que en el trabajo con los perceptores se intentó introducir una perspectiva histórica a modo de relato de vida que ofreciera luces sobre la influencia de las actuaciones políticas concretas sobre sus sucesos vitales. Esto explica que en la propuesta 
metodológica que aquí se presenta, la perspectiva de los perceptores no sea incorporada, y se centre solo en la comparativa entre políticos y técnicos, ya que la forma de recoger sus discursos ha sido diferente y, por tanto, no susceptible de ser analizada desde los mismos parámetros que la de los políticos y técnicos (con los que sí se usó el mismo esquema en la obtención de la información). Se es consciente de la importancia y la centralidad de la percepción de los perceptores para el ámbito de las políticas sociales, pero la misma ha sido ya tenida en cuenta en otros productos científicos. Además, más allá de la dificultad metodológica encontrada de partida por no usar la misma estructura en la construcción de los grupos de discusión y entrevistas (una cuestión central para el análisis comparativo del léxico) la finalidad de este artículo no es tanto el análisis de las políticas sociales como la oportunidad de usar la metodología que aquí se desarrolla, y así ha quedado reflejado en el apartado introductorio.

En relación al trabajo de campo hecho con los dos colectivos cuya comparativa se muestra en este artículo (políticos y técnicos), los informantes de las entrevistas en profundidad fueron seleccionados mediante muestreo cualificado (Coller, 2005).

Se llevaron a cabo un total de 5 entrevistas en profundidad, siendo la duración media de las mismas de 45 minutos. A esto habría que añadir los tres grupos focales, con una duración aproximada de $1 \mathrm{~h} 30$. Primero se hicieron todas las entrevistas y posteriormente, una vez hechas y transcritas, se llevaron a cabo los grupos focales, como forma de poder ajustar e introducir aspectos que en las entrevistas no fueron abordados. Se ha de aclarar que, principalmente para el caso de los perfiles político, las personas a las que se ha accedido han sido perfiles de primer nivel en el ámbito de las decisiones en las políticas autonómicas, de tal forma que aun siendo consciente del escaso número de "voces", son de un alto nivel de calidad y representatividad por el papel desempeñado.

Se adjunta a continuación una tabla descriptiva de los perfiles participantes en la investigación, así como el tipo de herramienta desarrollada, correspondiendo las siglas GF a "grupo focal" y EP a "entrevista en profundidad".

Tabla 1: Perfiles participantes políticos y técnicos de la investigación y herramientas utilizadas.

\begin{tabular}{|l|l|c|}
\hline ID & Perfil del grupo & Participantes \\
\hline GF1 & $\begin{array}{l}\text { Técnicos de entidades de servicios sociales (públicas y } \\
\text { tercer sector) }\end{array}$ & 7 \\
\hline GF2 & Técnicos de servicios de empleo (públicas y tercer sector). & 7 \\
\hline GF3 & Responsables políticos & 3 \\
\hline EP1 & Responsable político (sector público) & 1 \\
\hline EP2 & Responsable político (tercer sector) & 1 \\
\hline EP3 & Técnico (sector público) & 1 \\
\hline EP4 & Técnico (sector público) & 1 \\
\hline EP5 & Técnico 3 (tercer sector) & 1 \\
\hline
\end{tabular}


En el trabajo de campo se ha seguido una estrategia de entrevista estandarizada no programada (Denzin, 1970) o entrevistas conversacionales, en donde la estandarización vendría por la focalización en un mismo conjunto de información; permitiéndose al entrevistador adaptar la formulación y el orden de las preguntas a cada entrevistado.

A la hora de diseñar el guion de preguntas, decidimos seguir el esquema de Kvale (1996), que distingue entre preguntas temáticas de investigación (PTI) conformando éstas los grandes ejes temáticos sobre los que queríamos indagar, y las preguntas dinámicas de entrevistas (PDE), consistentes en la traducción de esos grandes ejes a un lenguaje más concreto que pueda ser usado y entendido por el entrevistado en el desarrollo de la entrevista. A una pregunta temática de investigación pueden corresponderle varias preguntas dinámicas posibles como puede apreciarse en el ANEXO 1.

El guion utilizado ha sido el mismo tanto para las entrevistas en profundidad como para los grupos focales con el sector político y con el sector técnico, llevando a cabo una adaptación del orden en función de cómo iban surgiendo los temas (sobre todo en los grupos focales). El uso de ambas técnicas nos permitía una forma muy complementaria de recogida de información, ya que el grupo focal aportaba a la entrevista la posibilidad de recoger el contraste de discursos y percepciones. Tanto las entrevistas, como los grupos focales fueron grabados y transcritos de acuerdo con el consentimiento informado de todos los participantes. Los datos personales de los participantes, así como cualquier elemento identificador, fueron sistemáticamente anonimizados en los materiales finales.

\subsection{Preparación de la información para el trabajo con el software Iramuteq.}

Una vez obtenido todo el material transcrito pasamos a la preparación del mismo para poder ser analizado desde el software Iramuteq. La apuesta por el uso de este software, a diferencia de otros posibles, vino determinada, en primer lugar, por ser una herramienta conocida por algunos miembros del equipo, al haber sido usada en otros proyectos anteriormente. Por otro lado, su uso y descarga es gratuita, a lo que se le sumaba que daba respuesta a esa primera parte de un análisis más cuantitativo-estadístico del léxico, para pasar, con posterioridad, a esa segunda parte de análisis cualitativo-relacional. Es importante de partida aclarar que el uso de esta herramienta por si sola carecería de sentido analítico, de ahí la importancia de que esta herramienta siempre se use como forma preliminar para una primera aproximación, pero después ha de ser complementada con otro tipo de técnicas de investigación que nos ayuden a profundizar más en los fenómenos abordados.

Para ello lo primero que se hizo fue crear un solo archivo de Word con las transcripciones de los 3 grupos focales (GF1-GF3) y las 5 entrevistas en profundidad (EP1-EP5). Cada una de las transcripciones de las entrevistas y de los grupos focales fueron convertidas en un subcorpus textual definido por 
una variable categórica, considerada metadatos. Las variables categóricas son "conjuntos de datos que pueden clasificarse en un número limitado de valores o categorías distintas, definidos sobre la base de alguna característica cualitativa" (Molina, 2017:7). La elección de estas variables depende de los intereses de la investigación e implica la selección de textos en base a este tipo de variables, por la voluntad de estudiar las configuraciones textuales de las diferentes categorías que la definen, sobre una misma temática de estudio. En nuestro caso estas variables fueron dos: perfil político o perfil técnico.

La forma de ir configurando estos subcorpus por variables categóricas es la introducción de cuatro asteriscos $(* * * *)$. Luego se introducen las variables precedidas de un asterisco, seguido de un guion bajo (_) y el código del valor de la variable. Se pueden introducir nuevas variables añadiendo un espacio y repitiendo la secuencia. De esta forma el corpus textual quedó codificado de la siguiente forma:

Tabla 2: Codificación del corpus textual para el software Iramuteq.

\begin{tabular}{|l|l|}
\hline ID perfil de EP y GF & Cofidicación Iramuteq \\
\hline $\begin{array}{l}\text { GF1: Técnicos de entidades de servicios sociales } \\
\text { (públicas y tercer sector) }\end{array}$ & $* * * * * G F \_1 *$ perfil_tec \\
\hline $\begin{array}{l}\text { GF2: Técnicos de servicios de empleo (públicas y } \\
\text { tercer sector). }\end{array}$ & $* * * * * G F \_2 *$ perfil_tec \\
\hline GF3: Responsables políticos & $* * * * * G F \_3 *$ perfil_pol \\
\hline EP1: Responsable político (sector público) & $* * * * * E P \_1 *$ perfil_pol \\
\hline EP2: Responsable político (tercer sector) & $* * * * * E P \_2 *$ perfil_pol \\
\hline EP3: Técnico (sector público) & $* * * * * E P \_3 *$ perfil_tec \\
\hline EP4: Técnico (sector público) & $* * * * * E P \_4 *$ perfil_tec \\
\hline EP5: Técnico 3 (tercer sector) & $* * * * * E P \_5 *$ perfil_tec \\
\hline
\end{tabular}

Una vez codificado el archivo Word con estas claves se pasó a revisar el texto siguiendo las siguientes indicaciones para asegurar así que el software Iramuteq funcionaría correctamente (Molina, 2017):

- Eliminación del texto producido por el investigador (como las preguntas realizadas en el marco de las entrevistas y los grupos focales).

- No justificación del texto.

- No resaltar ninguna palabra con negrita, cursiva, subrayado o similar.

- Utilizar siempre las mismas siglas en el caso de que se hayan usado.

- Utilizar formato numérico para los números.

- No utilizar caracteres especiales como comillas, apóstrofes, símbolos y asteriscos dentro del texto.

Tras este trabajo de formato del archivo en Word se convirtió el mismo en formato UTF_8 (.txt). En este caso, se usó para esta conversión el programa No- 
tepad+++. Finalmente, el archivo ya estaba preparado para ser introducido en el programa y ser explotado y analizado por el mismo.

\section{RESULTADOS.}

\subsection{Análisis de frecuencias}

Se presenta a continuación un resumen general que arroja el programa Iramuteq de toda la información cualitativa obtenida en la fase de trabajo de campo con un total de 3 grupos focales y 5 entrevistas en profundidad.

El texto total analizado ha sido de 59303 palabras (ocurrencias) divididas en 8 textos (los correspondientes a las trascripciones de los 3 grupos focales y las 5 entrevistas en profundidad). Del total de palabras, 3126 son conjuntos de palabras diferenciadas y aglutinadas en formas (sustantivos, verbos, adjetivos...) mientras que 1334 son palabras que aparecen una sola vez a lo largo de todos los textos (hápax)

Tabla 3. Resumen estadístico

\begin{tabular}{|l|l|}
\hline Número de textos & 8 \\
\hline Número de ocurrencias & 59303 \\
\hline Número de formas & 3126 \\
\hline Número de hápax & 1334 \\
\hline Media de ocurrencias por textos & 7412,87 \\
\hline
\end{tabular}

En la siguiente gráfica se presenta este resumen de tal forma que en el eje de abscisa quedan representados los logaritmos de rangos y en el eje de ordenadas quedan representados los logaritmos de frecuencias. 


\section{Gráfica 1: Análisis de frecuencias}

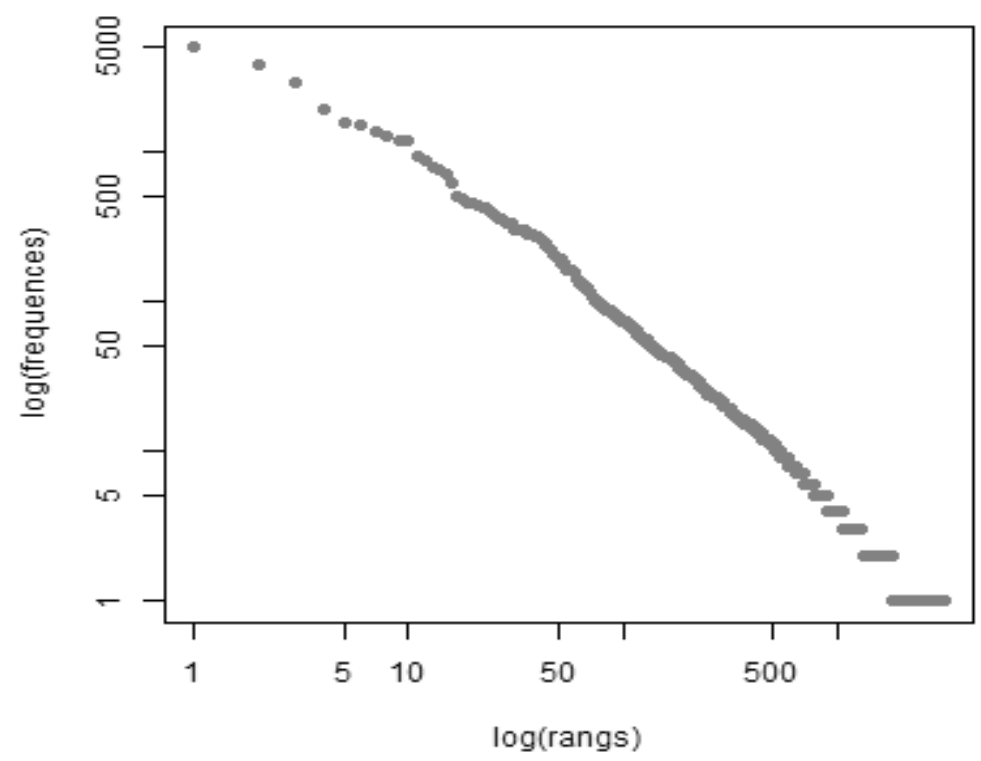

En este análisis preliminar se puede diferenciar también entre las formas activas que aparecen en el texto (que son las que se considerarán posteriormente en el análisis) y las formas suplementarias, que no serán consideradas para el análisis y que son principalmente conectores (artículos, conjunciones, preposiciones ...). En este sentido, el el software te da la opción del marcar las normas para la "limpieza del texto", lo que resulta muy importante para una correcta interpretación de los resultados.

En la tabla 2 podemos observar las 20 formas activas más utilizadas en el texto y la frecuencia con la que aparecen. 
Tabla 4. Principales formas activas presentes

\begin{tabular}{|l|l|l|}
\hline & FORMAS & FRECUENCIAS \\
\hline 1 & decir & 427 \\
\hline 2 & ir & 399 \\
\hline 3 & social & 351 \\
\hline 4 & hacer & 345 \\
\hline 5 & más & 332 \\
\hline 6 & empleo & 302 \\
\hline 7 & bueno & 300 \\
\hline 8 & mucho & 297 \\
\hline 9 & crearlcreer & 272 \\
\hline 10 & persona & 253 \\
\hline 11 & ver & 209 \\
\hline 12 & político & 206 \\
\hline 13 & servicio & 199 \\
\hline 14 & trabajar & 194 \\
\hline 15 & claro & 189 \\
\hline 16 & ahora & 182 \\
\hline 17 & gente & 181 \\
\hline 18 & trabajo & 168 \\
\hline 19 & administración & 164 \\
\hline 20 & tema & 163 \\
\hline
\end{tabular}

\subsection{Análisis de especificidades}

Aunque anteriormente se ha visto un análisis global de todo el texto, se pasa ahora a su análisis en función de las variables utilizadas para lo que se lleva a cabo un Análisis de Especificidades. Este tipo de análisis asocia los textos del corpus a variables de sus productores, previamente definidos por los investigadores y muestra una comparativa del uso de formas específicas (Molina, 2017). En este caso se han definido dos variables que son las que interesa comparar, perfil político (*perfil_pol) y perfil técnico (*perfil_tec).

A parte de estas dos variables, se han definido 7 grandes categorías conceptuales cada una de las cuales, a su vez, está compuesta por una serie de formas que aparecen en los discursos analizados. Estas categorías conceptuales se han definido partiendo de su consideración como ejes conceptuales en los discursos sobre las políticas de inclusión social. 
Tabla 5: Categorías conceptuales y formas correspondientes

\begin{tabular}{|c|c|c|}
\hline & CATEGORIA CONCEPTUAL & FORMAS \\
\hline 1 & CONCEPTOS & $\begin{array}{l}\text { Inclusión } \\
\text { Pobreza } \\
\text { Solidaridad } \\
\text { Bienestar } \\
\text { Exclusión }\end{array}$ \\
\hline 2 & DERECHOS & $\begin{array}{l}\text { Empleo } \\
\text { Derecho } \\
\text { Vivienda } \\
\text { Educación } \\
\text { Salud }\end{array}$ \\
\hline 3 & HERRAMIENTAS DE INTERVENCIÓN & $\begin{array}{l}\text { Recurso } \\
\text { Prestación } \\
\text { Salario } \\
\text { Subvención }\end{array}$ \\
\hline 4 & HERRAMIENTAS DE PROGRAMACIÓN & $\begin{array}{l}\text { Plan } \\
\text { Estrategia } \\
\text { Programa } \\
\text { Itinerario }\end{array}$ \\
\hline 5 & ACTORES & $\begin{array}{l}\text { Administración } \\
\text { Entidad } \\
\text { Empresa }\end{array}$ \\
\hline 6 & ACCIONES & $\begin{array}{l}\text { Intervención } \\
\text { Transformación } \\
\text { Intermediación } \\
\text { Información } \\
\text { Orientación }\end{array}$ \\
\hline 7 & DISTRIBUCIÓN COMPETENCIAL & $\begin{array}{l}\text { Ayuntamiento } \\
\text { Consejería } \\
\text { Estado }\end{array}$ \\
\hline
\end{tabular}

Una vez definidas previamente estas categorías conceptuales con sus formas respectivas, interesaba analizar la presencia que éstas tenían en los discursos de los diferentes actores con los que se ha trabajado: políticos y técnicos. Para ello se ha llevado a cabo un análisis de frecuencias relativas de formas (frecuencia absoluta partido por el total de la muestra). Es una frecuencia que no está afectada por el tamaño de la muestra, por tanto, permite la comparación de como unas formas y otras aparecen presentes en los discursos de políticos y técnicos. Estas frecuencias están expresadas en tantos por mil. 
A continuación, mostramos los diferentes gráficos resultantes de este análisis de especificidades por variables (*perfil_pol y *perfil_tec) y las categorías conceptuales diseñadas con sus respectivas formas.

Grafica 2: Análisis de especificidades por variables y categorías conceptuales.

\section{Gráfica 2.1 Conceptos}

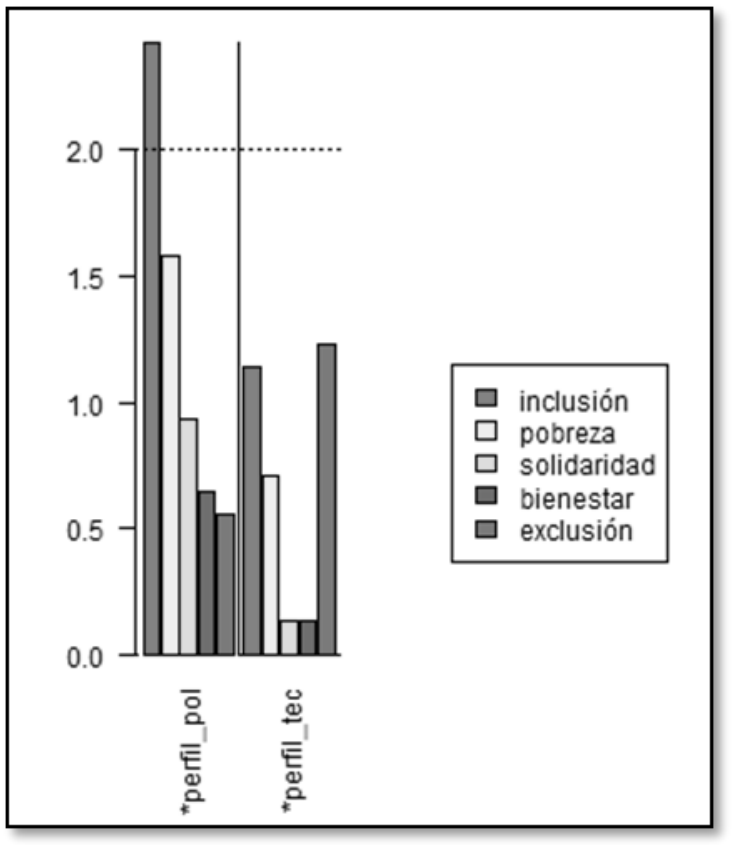

Los grandes conceptos (Gráfica 2.1.) que enmarcan las políticas de inclusión social aparecen mucho más presente en el discurso político que en el técnico, destacando por encima de todo el discurso sobre el propio concepto de "inclusión". Por el contrario, en el discurso técnico estos grandes conceptos aparecen mucho menos presentes y casi al mismo nivel discursivo aparecen "inclusión" / "exclusión", siendo conscientes los técnicos que han de trabajar con la presencia de este binomio, sin negar, en el discurso, la máxima sobre la que deben luchar las políticas de inclusión so-

cial: la exclusión social. Sorprende como los políticos usan en mayor medida el concepto de "pobreza", un concepto mucho más reduccionista y que da menos cobertura a la complejidad de situaciones que se deben abordar desde las políticas de inclusión social. Conceptos más generales como "bienestar" o "solidaridad" se hacen casi inexistentes en el discurso técnico, estando, por tanto, estos conceptos mucho más arraigados en el discurso ideático de los políticos. 
Gráfica 2.2 Derechos

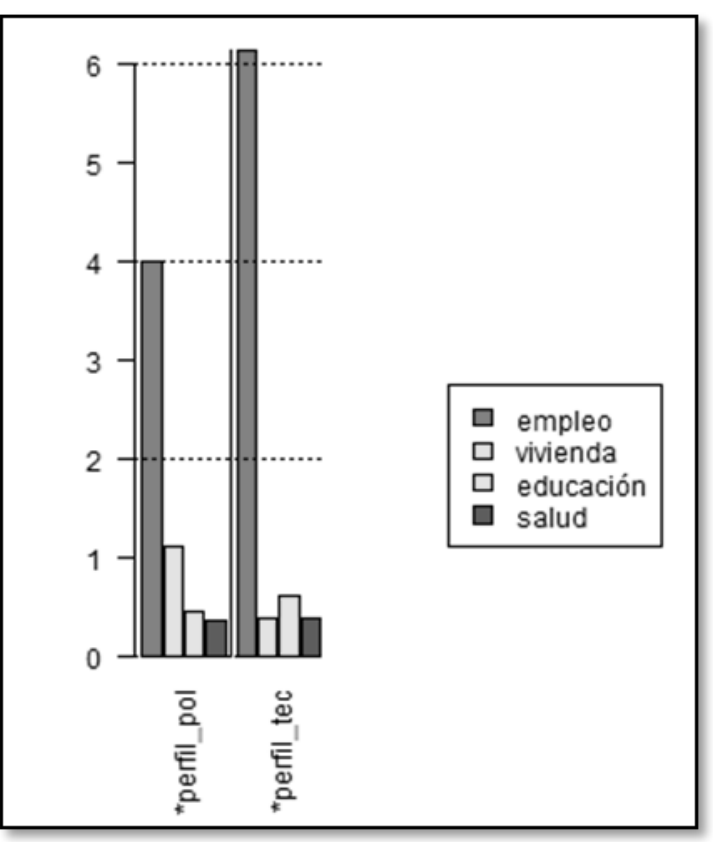

Analizar la presencia de derechos (Gráfica 2.2) en los discursos sobre las políticas de inclusión social se entiende fundamental para entender la concepción de estas políticas, o bien como algo subsidiario solo relacionado con "lo social" o como algo mucho más amplio y global que debe abarca muchos otros derechos. En este sentido, el discurso político y técnico es muy parecido, estando el "empleo" como eje vertebrador que ha de enmarcar cualquier política de inclusión social, y, de forma subsidiaria, otros derechos. De aquí se puede derivar la concepción de que la inclusión pasa necesariamente por el empleo, afirmación esta, que está siendo muy cuestionada en los últimos tiempos por el tipo de empleo que se está generando, un empleo precario, inestable, y que, muchos informes están arrojando, no garantiza que la gente pueda salir de la vulnerabilidad y la pobreza, generándose así el fenómeno de los "trabajadores pobres" (Informe Foessa, 2019) 


\section{Gráfica 2.3 Herramientas de Intervención}

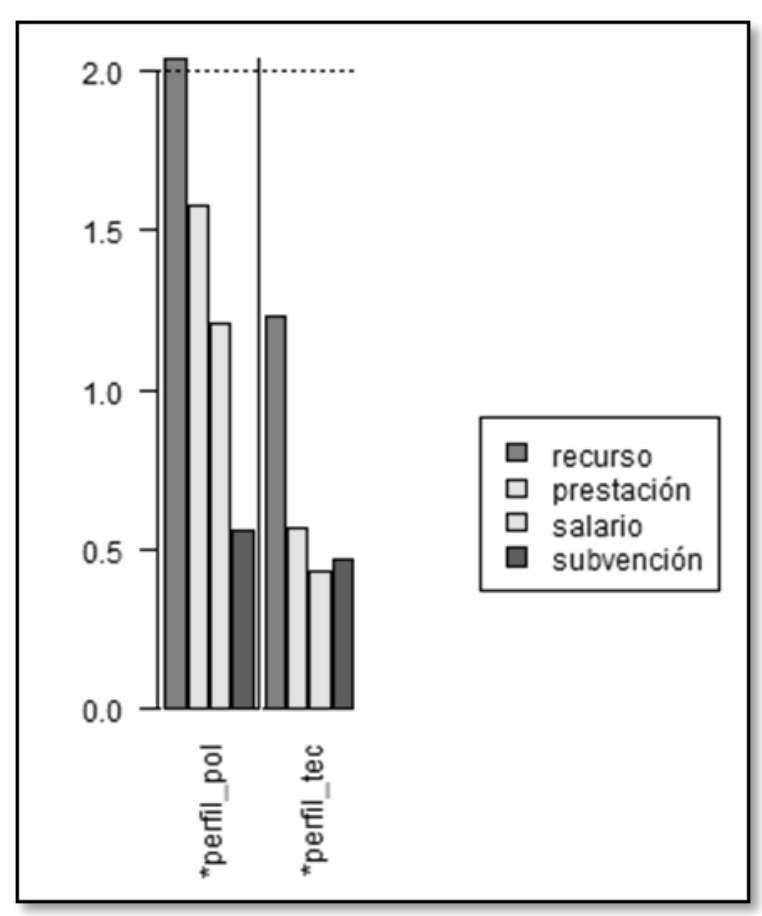

En cuanto a las herramientas (Gráfica 2.3) con las que se cuenta para llevar a cabo la intervención desde las políticas de inclusión social, el concepto "recurso" es el más presente en el ámbito discursivo en ambos perfiles, lo cual deja entrever la distancia entre el discurso en la categoría conceptual "Conceptos" y esta categoría conceptual, en la medida que el "recurso" es algo muy reduccionista si hablamos de inclusión social. En el discurso político aparecen términos más diversificados que aluden de forma más concreta a la dimensión material (económica) del recurso como "prestación"

o incluso "salario" aludiendo al Programa de Garantía de Ingresos Mínimo de la Junta de Andalucía. Sea como fuere, el peso de lo económico sigue estando muy presente cuando se habla de intervención desde las políticas de inclusión social. 


\section{Gráfica 2.4. Herramientas de Programación}

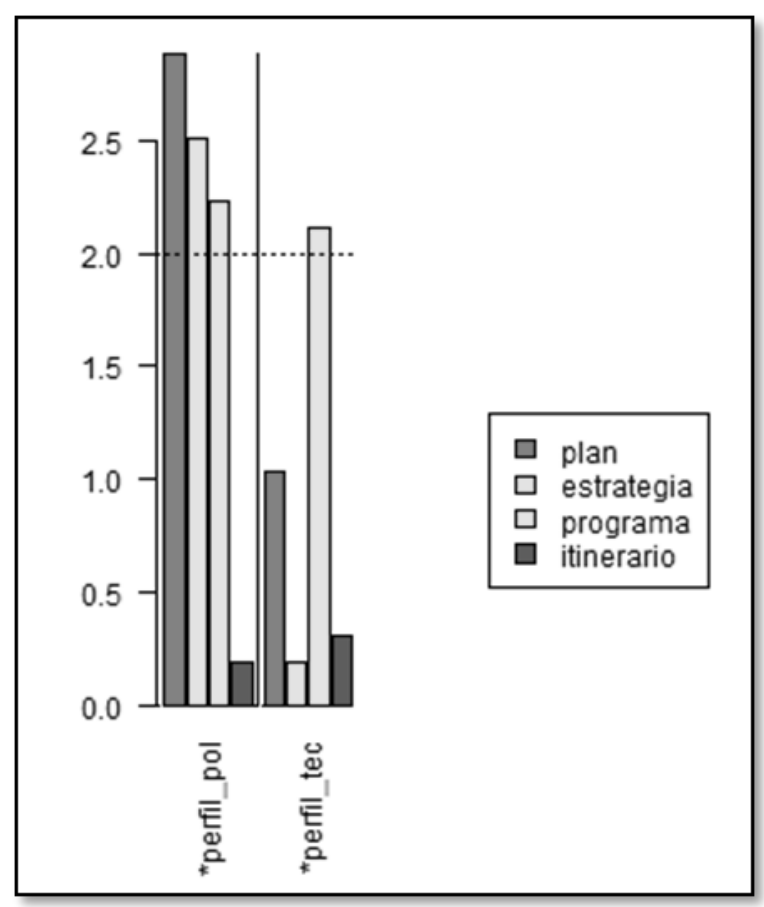

En lo relativo al discurso de programación (Gráfica 2.4) de las políticas de inclusión social de nuevo se observa una distancia en los discursos de ambos perfiles. Aunque los dos manejan con la misma intensidad el concepto "programa", quizás porque es el concepto más extendido a nivel de programación, los políticos son mucho más tendentes al uso de conceptos que hacen alusión a una programación a gran escala ("plan" y "estrategia"), cuestión esta, que tiene pleno sentido en función al ámbito en donde ellos se ubican en relación a las políticas de inclusión social (ámbito del diseño y no de la ejecución). Pero mientras que el discurso político si tiene correspondencia con la terminología a la hora de hablar de herramientas de programación de las políticas de inclusión social, no es así en el caso de los técnicos, cuyo ámbito discursivo se queda principalmente enclavado en el término "programa" (y en menor medida en el término "plan") pero apenas aparece presente en su discurso el concepto "itinerario" que a nivel programático habría de ser la principal herramienta de programación en la intervención concreta con las personas destinatarias de esas políticas. 


\section{Gráfica 2.5 Actores}

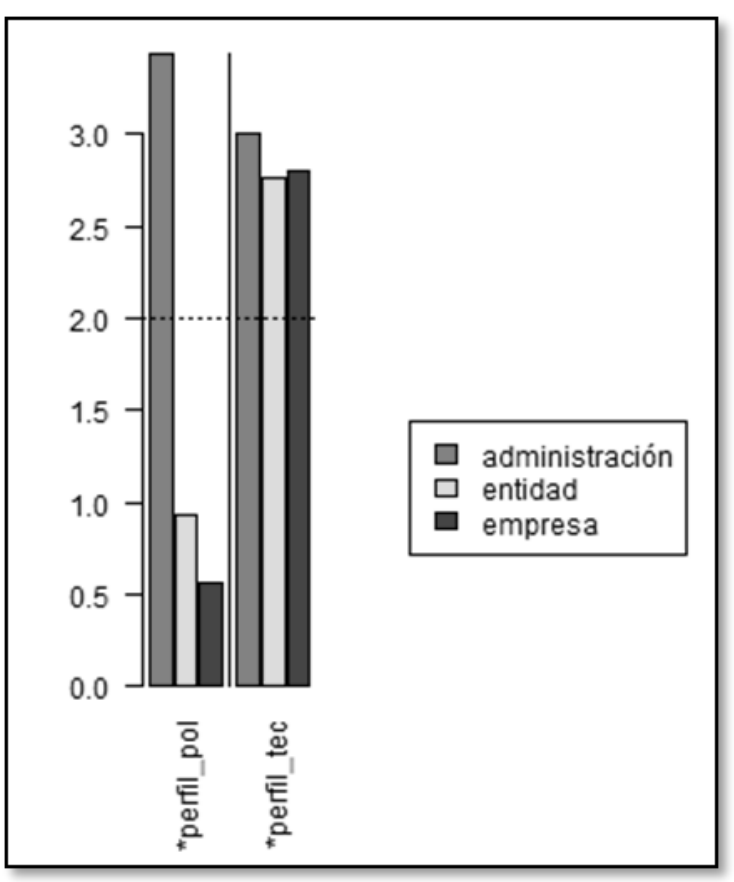

El análisis discursivo sobre quienes han de ser los actores (Gráficas 2.5) de las políticas de inclusión social si muestra una clara diferencia entre políticos y técnicos. Mientras que para los políticos existe una prominencia de la administración como principal actor de las políticas de inclusión social, en el caso de los técnicos, los actores, incluso a niveles muy parecidos en la presencia discursiva, han de ser, además de la administración, las entidades sociales y las empresas. De nuevo se puede observar la distancia entre un discurso basado en lo teórico (normativo) a un discurso basado en las praxis cotidianas, donde las políticas de inclusión social hoy en día están siendo ejecutadas y complementadas, en gran parte, por las entidades sociales y, en los últimos tiempos, también por las empresas, a través de sus políticas de Responsabilidad Social Corporativa. Muestra de ello es el papel que están jugando las fundaciones bancarias en la puesta en marcha de proyectos sociales dirigidos a los colectivos más vulnerables. 


\section{Gráfica 2.6. Acciones}

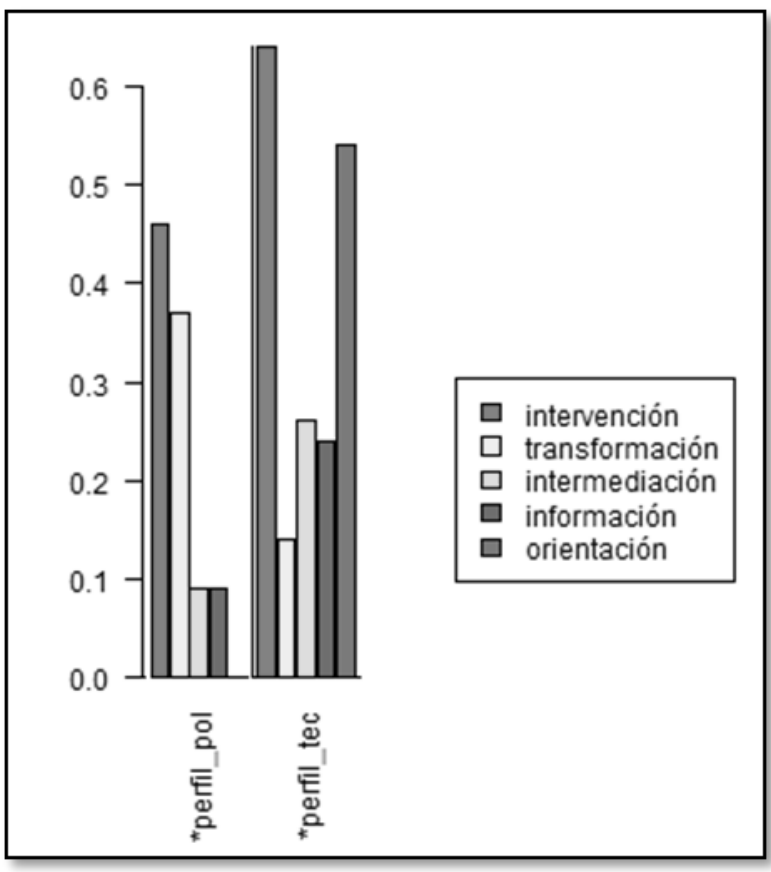

En cuanto a las acciones (Gráfica 2.6) que tienen cabida bajo el paraguas de las políticas de inclusión social, el concepto "intervención" es el más presente tanto en políticos como en técnicos, un concepto amplio en el que cualquier tipo de actividad tendría cabida. Pero más allá de ese concepto amplio aparecen posteriormente tipologías de acciones que reflejan perfectamente el tipo de contexto en el que ellos transitan por las políticas de inclusión social. Mientras que los políticos utilizan términos como "transformar", los técnicos diversifican más la terminología en la tipología de acciones describiendo así de una forma más detallada cuales son las acciones que hacen en su día a día. Así aparecen más presentes acciones del tipo "informar", "intermediar" o incluso un tipo de actividad que se hace muy presente en su discurso como es "orientar", actividad esta que ni siquiera aparece en el discurso político. 


\section{Gráfica 2.7 Distribución competencial}

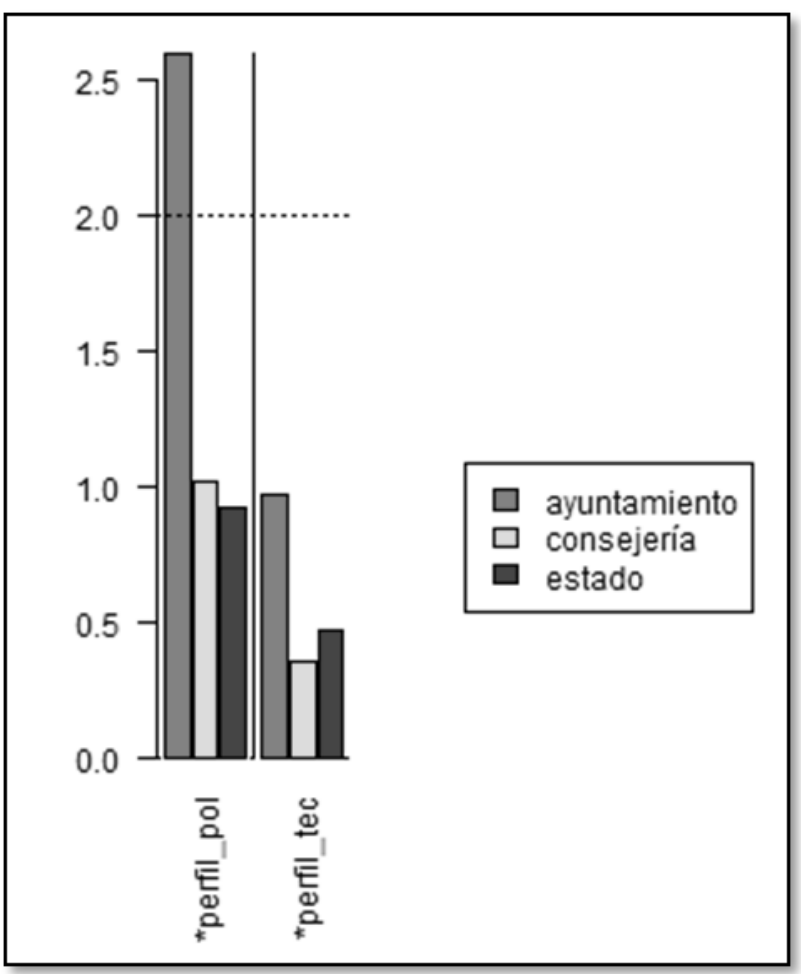

Por último, en relación a la última categoría conceptual relacionada con la distribución competencial (Gráfica 2.7) entre las diferentes administraciones en materia de políticas de inclusión social, de nuevo encontramos diferencias entre las variables analizadas. Mientras que en el discurso político el Ayuntamiento adquiere un protagonismo central, en el discurso técnico los tres niveles de administración (Estado, Consejería y Ayuntamiento) aparecen con una presencia muy similar. En este sentido se entiende que los políticos tienen un visión mucho más general y amplia sobre la financiación de estas políticas y, en sus discursos, queda reflejado como, finalmente, son los Ayuntamientos los que están marcando las diferencias en sus apuestas, o no, por dotar presupuestariamente las políticas de inclusión social que reciben los ciudadanos, siendo conscientes que las aportaciones de los niveles autonómicos y estatales no terminan siendo suficientes.

\subsection{Análisis de similitudes}

Este tipo de análisis que se presenta ahora se centra en analizar la conexión que existe dentro del corpus textual. Para ello identifica núcleos semánticos detectados por concurrencias entre palabras según sus conexiones en el texto (Camargo y Justo, 2013) ayudando, así, a identificar la estructura del contenido del corpus textual gracias a su visualización a modo de gráfico con forma de árbol (Moreno, 2017). A la hora de la interpretación del mismo se han de considerar las formas, que se encuentran en los nodos de la gráfica y los enlaces, que representan la co-ocurrencia entre ellos. A mayor frecuencia de las palabras, mayor 
tamaño de las mismas en el gráfico. A mayor co-ocurrencia entre palabras, más grueso se representa el enlace entre ellas (Ruiz Bueno, 2017).

Siguiendo con la finalidad de este artículo en la comparativa entre los discursos políticos y técnicos sobre las políticas de inclusión social en Andalucía, hemos hecho un análisis de similitud por cada una de las dos variables (*perfil_pol y *perfil_tec) que pasamos a comentar y analizar a continuación.

\section{Gráfica 3. Análisis de similitudes perfil político.}

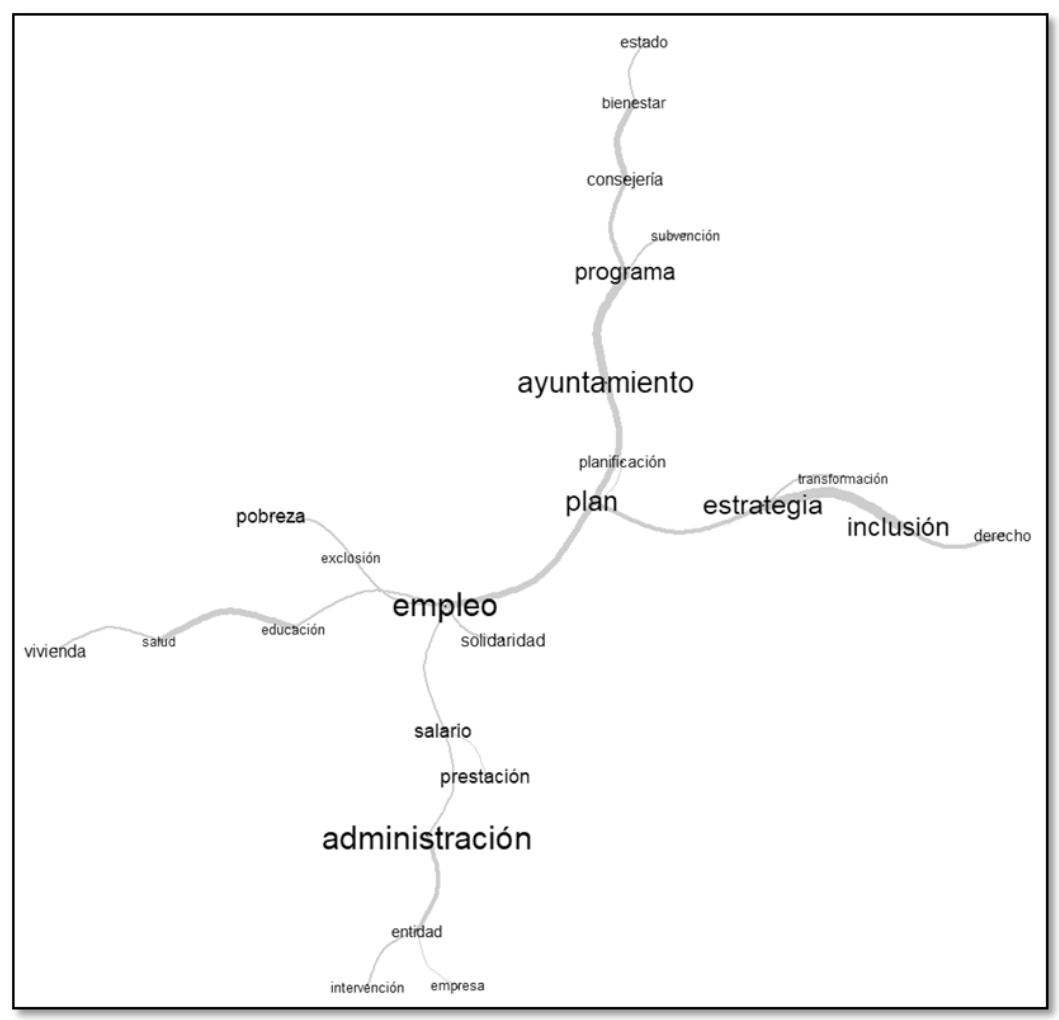

Los tres nodos centrales en el discurso político se encuentran representados por las formas lexicales "empleo" (que aparece en el centro), "ayuntamiento" y "administración" (las tres aparecen representadas con el mismo tamaño). De esta forma se refuerza así la idea de que las políticas de inclusión social pasan necesariamente por el "empleo" (idea esta que ya ha aparecido reflejada en los análisis anteriores). Igualmente, para los políticos el "ayuntamiento", a nivel competencial, ha de tener un papel central en este tipo de políticas, y a nivel de actores, es la administración (por encima de las entidades o las empresas) la que aparece con más fuerza en su discurso. 
En un segundo lugar estarían los nodos representados por las formas "plan", "estrategia" e "inclusión", dando muestra así del nivel más generalista y de planificación de las políticas de inclusión social que muestra el perfil político en sus discursos.

Como ya hemos comentado anteriormente, este análisis de similitudes también muestra las conexiones discursivas representadas por las líneas del árbol que une a las palabras y por el grosor de las mismas. Vemos como aparecen enlazadas "plan", "estrategia" e "inclusión" siendo muy fuerte el nexo de estas dos últimas formas. Esto da respuesta a la reciente aprobación de la "Estrategia Regional para la Cohesión y la Inclusión Social" en Andalucía (BOJA nº 172 de 5/09/18) que se presenta como la principal apuesta de política social para la inclusión social, de ahí que aparezca tan presente en el discurso del perfil político.

Otra conexión discursiva a resaltar es la establecida por las formas "programa", "ayuntamiento", "plan" y "empleo", reforzándose así, de nuevo, la importancia que el discurso político le da a las políticas locales en lo relacionado con la puesta en marcha de programas o planes específicos en materia de empleo.

Por último, se puede resaltar algunas de las conexiones periféricas del gráfico que aúna formas lexicales relacionadas como las que tienen que ver con los derechos ("vivienda", "salud" y "educación") o la que tiene que ver con los actores de las políticas de inclusión social ("administración", "entidades" y "empresa"). 
Gráfica 4. Análisis de similitudes perfil técnico.

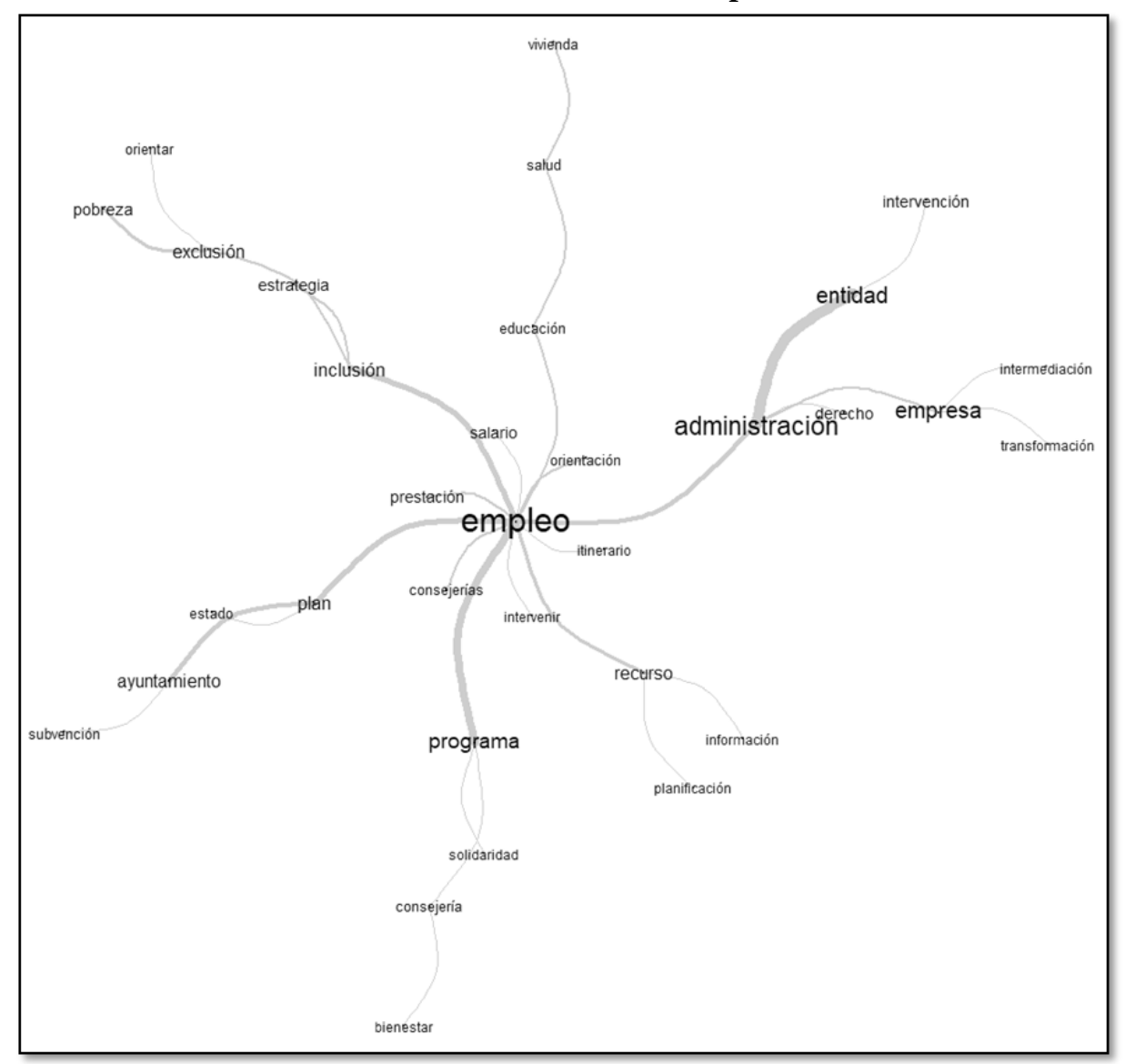

Al igual que pasaba con el Análisis de similitudes de perfil político, la centralidad la vuelve a tener la forma "empleo" muy por encima del resto de formas, que son más diluidas y minoritarias que en el perfil anteriormente analizado. También para los técnicos el empleo ha de ocupar un papel central en las políticas de inclusión social, mediante un formato de "programa", forma ésta con la que se une fuertemente en el gráfico.

Destaca, en un segundo momento, la centralidad en el discurso de los técnicos de la actoría en las políticas de inclusión social, apareciendo las formas "administración","entidad" y "empresa" con el mismo tamaño de representación y conectadas entre si, aunque la conexión entre las dos primeras es mucho más gruesa y fuerte que con la "empresa", reflejando, de esta forma, el discurso técnico, la realidad actual en las relaciones entre los actores de las políticas de inclusión social. 
Por último, en relación a las conexiones más periféricas de este gráfico, resaltar la línea que une los grandes conceptos de las políticas sociales ("inclusión", "exclusión" y "pobreza"), vinculados, a su vez, en la misma línea, con la forma "estrategia", reforzándose así lo ya planteado anteriormente en relación a la reciente aprobación de la "Estrategia Regional para la Cohesión y la Inclusión Social". Igualmente destacar la línea periférica que une las formas relacionadas con los derechos sociales ("empleo", "educación", "salud" y "vivienda") reforzándose así el discurso técnico de que las políticas de inclusión no sólo tienen que ver con "lo social".

\subsection{Clasificación Jerárquica Descendente}

Este análisis se basa en el método Reinert que parte de la premisa de que las palabras no son independientes unas de otras, sino que reflejan temas subyacentes. La tesis principal de Reinert $(1987,1998,2003)$ es que todo discurso se expresa a partir de un conjunto de palabras que constituyen unidades de significado de manera independiente a su construcción sintáctica. Estas unidades de significado evocan una forma de pensar sobre el objeto del que se habla, un campo de pensamiento, pues, a partir de ellas, los enunciados adquieren sentido.

La redundancia de sucesiones de palabras, o la concatenación de palabras que componen un discurso determinado, permite localizar los "mundos lexicales" evocados por los enunciadores. Es por ello que, como plantea Moreno (2017:4) "el análisis estadístico de los discursos cobra su sentido, pues permite localizar repeticiones frecuentes de léxicos, que tienen una organización habitual, para referirse a un mismo objeto". En concreto, se analiza la organización y la distribución de las palabras principales co-ocurrentes en los enunciados simples de un texto, es decir, la presencia simultánea de varias palabras funcionales (sustantivos, verbos y adjetivos) en un mismo enunciado. Al utilizar un vocabulario determinado, el locutor convoca un "lugar" de enunciación, el cual se define por oposición a otros lugares (Alba, 2004).

La propuesta de Reinert (1983), siguiendo un análisis “clúster", permite clasificar los datos en grupos (Kaufman y Rouseeeuw, 2009) con la característica de homogéneos entre los elementos que lo conforman, pero, a la vez, los más diferente a otros grupos. Se define como "descendente" porque va de arriba abajo, es decir, su punto de partida es un solo grupo que incluye a todos los individuos, y en cada etapa realiza las subdivisiones en grupos/clases.

Una vez aclarada la metodología de análisis de este apartado se introduce en la herramienta Iramuteq, una vez más, el material léxico de la investigación. En esta ocasión partimos de todo el conjunto del discurso sin distinción por variables (*perfil_pol y *perfil_tec) ya que interesaba conocer cuáles eran aquellos clústeres que se generaban de todo el discurso sobre políticas de inclusión social, y en un segundo momento, se analizaría cuales eran los más presentes en una y otra variables, a través de la comparativa con el Análisis de Especificidades por variables y categorías conceptuales (Gráficas 2). 
El análisis arroja la existencia de 4 clúster en el discurso analizado. Tras analizar el contenido de las formas asignadas se le ha otorgado un concepto a cada uno de ellos, resultando el siguiente gráfico. Se ha de aclarar que no son categorías "puras", de tal forma que en cada uno de los clúster puede haber presencia de formas que cabrían en algunos de los otros clúster generados.

\section{Gráfica 5: Dendograma de formas de análisis clúster}

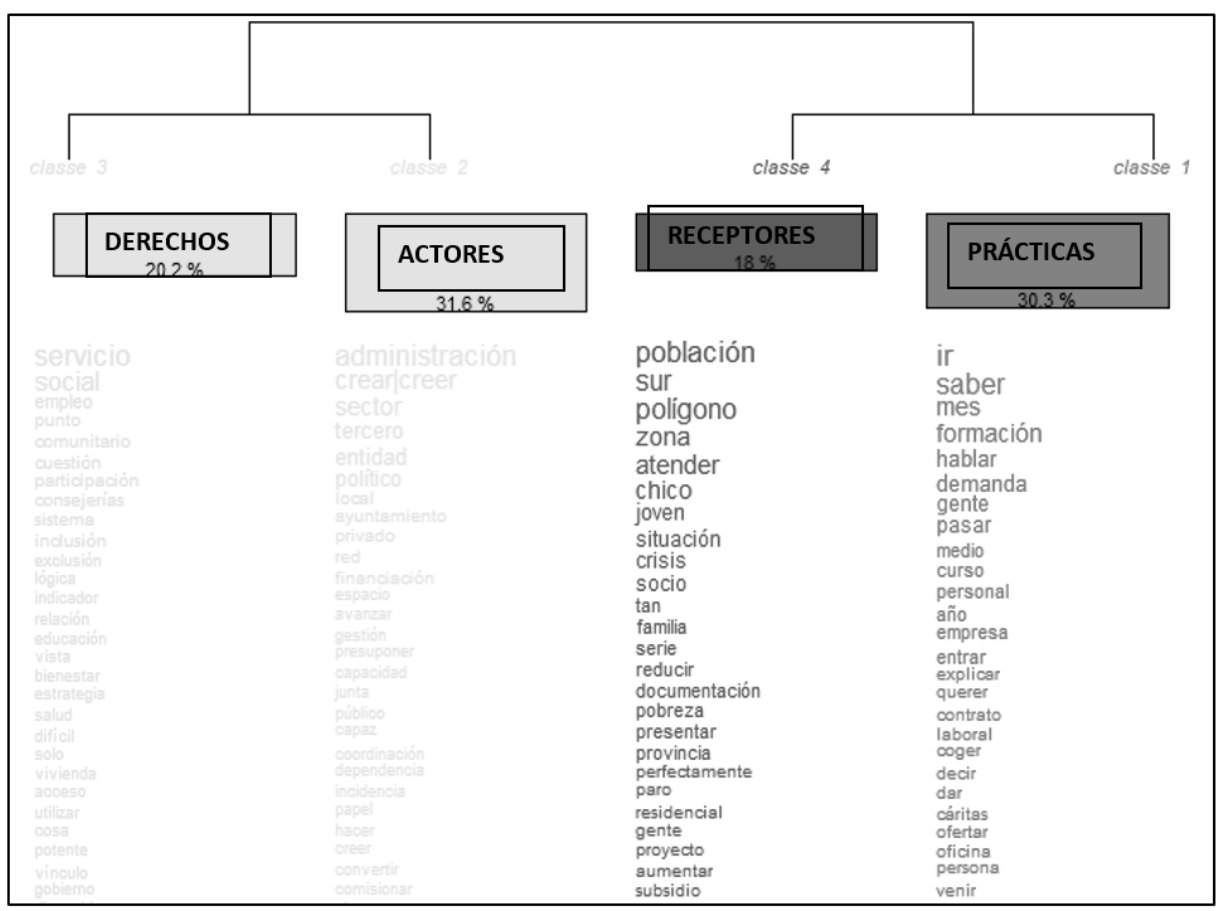

Clúster 1: PRÁCTICAS. Es de las temáticas más presentes en el discurso con una tasa de porcentaje de presencia de las formas que la componen de un $30,3 \%$. Este clúster aglutina el léxico que tiene que ver con las praxis cotidianas de la intervención social que se genera en el marco de las políticas de inclusión social. Este clúster aparece mucho más presente en el discurso de la variable (*perfil_tec).

Clúster 2: ACTORES. Es la temática con más presencia en el discurso de políticos y técnicos por encima del resto de clúster (31,6\%). El mismo aglutina todo lo relacionado con los actores de las políticas de inclusión social, así como lo relacionado con los niveles competenciales. Esta temática aparece presente en ambas variables analizadas (*perfil_pol y *perfil_tec), aunque tratado de forma muy diferente, tal y como se ha podido ver en el Análisis de Especificidades. 
Clúster 3: DERECHOS. Esta temática aglutina todo lo relacionado con los derechos sociales, así como los grandes conceptos vinculados al mundo de la intervención social y de las políticas de inclusión social con una tasa de porcentaje de presencia de las formas que la componen del 20,2\%. De nuevo este clúster se hace presente en ambas variables (*perfil_pol y *perfil_tec), aunque con mayores semejanzas entre unos y otros que en el clúster anterior.

Clúster 4: RECEPTORES. Esta es la temática menos presente en el discurso, con un $18 \%$ de presencia de sus formas léxicas. Aglutina lo relacionado con los destinatarios de las políticas de inclusión social y de la intervención social que ésta conlleva, no solo desde un sentido de colectivos concretos sino también desde un sentido territorial y conceptual. Este clúster aparece más presente en la variable (*perfil_tec) ya que, al hablar de receptores de políticas de inclusión social, son los técnicos los que están más próximos a ellos en su día a día y, por tanto, los que lo tienen más presentes en su imaginario y su discurso.

\section{CONCLUSIONES}

El trabajo plasmado a lo largo de este artículo permite afirmar que el análisis del léxico sistematizado, y volcado en ilustraciones gráficas, es una buena estrategia para avanzar, de una forma mucho más clarificadora, en las diferentes representaciones sociales que sobre un mismo objeto (en este caso una misma política de inclusión social) tienen los diferentes actores. Los resultados del análisis de los discursos han permitidos identificar la tipología de los mismos, y valorar el posicionamiento y relevancia que cada grupo de actores le otorga a una determinada temática. Además, ha permitido identificar cuáles eran los aspectos concretos en torno a los cuales giran los discursos de los actores, y, lo más relevante, identificar las relaciones entre las temáticas presentes. Para esto último, el Análisis de Similitudes como la Clasificación Jerárquica Descendente basada en la construcción de clúster entendemos que han sido muy oportunas y que aportan mucho al respecto.

Desde el simple análisis del léxico y posterior análisis del discurso podemos extraer esa doble visión que, sobre las políticas de inclusión social, muestran los diferentes actores: los que las diseñan (políticos) y los que las ejecutan (técnicos), reafirmando así esa idea de Cohen (1993) de que los modos de conocimiento son inextricables de los modos de identidad. Los primeros muestran un discurso arraigado en lo ideático, en lo generalista y, en muchas ocasiones, alejado de la praxis cotidiana, al contario que el discurso técnico, que muestra un claro conocimiento de lo cotidiano de la gestión de estas políticas (lo concreto). Ambos representan así, los diferentes conocimientos entre la realidad transitada y la realidad construida solo desde lo teórico (Purcell, 1998; Perkins, 1995; Morris y Bastin, 2004 y Ruiz y González, 2006). Esta doble visión revela la necesidad de intercambio, comunicación y complementariedad de ambas perspectivas en todos los análisis destinados a lograr y mejorar las políticas de inclusión social de una forma efectiva (Rosa y Encina, 2004; Jaraíz, 2011), ya que, como planteaba 
Fisher (2003) el cambio social y el cambio político frecuentemente se producen debido al conjunto de significados compartidos de conceptos y constructos.

A este respecto se defiende firmemente que este tipo de análisis y trabajo científicos pueden tener una aplicabilidad práctica muy interesante desde una vocación de investigación aplicada. El poder trabajar y devolver a los actores implicados los resultados de este análisis que aquí se presenta, daría lugar a una nueva fase de investigación-acción- participativa, donde se podría trabajar de forma conjunta este contraste de miras y percepciones sobre las políticas sociales, en pos de acercar ambos tipos de conocimientos y mejorar, en el fondo el diseño y la ejecución de las mismas.

Igualmente, se entiende que sería muy oportuno completar este análisis con el análisis del discurso del tercer actor central en las políticas de inclusión social, los perceptores de las mismas, ya que, de esta forma, se completaría mucho más la evaluación desde una perspectiva multiactor. Ya hemos explicado anteriormente las limitaciones para poder llevarlo a cabo en esta ocasión, pero se nos presenta como una línea a profundizar en investigaciones futuras.

Para terminar, creemos que es importante ser conscientes de la limitación de esta propuesta metodológica, y, por ello, la consideramos como un punto de partida que posteriormente deberá ser complementado con otro tipo de metodologías de análisis cualitativo más profundo, que permitan ahondar mucho más en el contenido de los discursos.

\section{BIBLIOGRAFÍA.}

ALBA, M. (2004): "El método Alceste y su aplicación al estudio de las representaciones sociales del espacio urbano: el caso de la ciudad de México", Papers on social representations, 13(1), pp.1-20.

BARDIN, L. (1996): Análisis de Contenido, Madrid, Akal.

BATHIA, V.K. (1993): Analysing genre: language use in professional settings, Londres, Longman Group UK.

BAZERMAN, C. y PARADIS, J. (Comps.) (1991): Textual Dynamics in the Professions: Historical and Contemporary Studies of Writing in Professional Communities, Madison, WI: University of Wisconsin Press.

BERELSON, B. (1952): Content Analysis in Communication Researches. Glencoe III, Free Press.

BERGER, P. Y LUCKMANN, T. (1995): La construcción social de la realidad, Buenos Aires, Amorrortu.

CAMARGO, B.V. y JUSTO, A.M. (2013) Tutorial para uso do software de análise textual IRAMUTEQ, disponible en http://www.iramuteq.org/documentation/fichiers/ tutoriel-en-portugais [consulta: 18-09-18]

CASTORIADIS, C. (1989): La Institución Imaginaria de la Sociedad, Buenos Aires, Tusquets Editores.

COHEN, A. P. (1993): "Segmentary knowledge: a Walshay sketch" en An antthropological critique of development, Londres: Routledge, pp. 136-152.

COLLER, X. (2005): Estudio de casos, Madrid, CIS. 
CONSEJERÍA DE IGUALDAD Y POLÍTICAS SOCIALES (2018): Estrategia Regional Andaluza para la Cohesión e Inclusión Social. Intervención en zonas desfavorecidas. (BOJA n ${ }^{\circ} 172$ de 5/09/18).

DENZIN, N.K. (1970): The research act, Chicago, Aldine.

FISCHER, F. (2003): Reframing Public Policy: Discursive Politics and Deliberative Practices, Oxford, Oxford University

FUNDACIÓN FOESSA (2019): VIII Informe Foessa sobre Exclusión y Desarrollo Social en España, Madrid, Fundación Foessa-Cáritas Española.

GUNNARSSON, B.L. (2000): "Análisis aplicado del discurso" en El discurso como interacción social, Barcelona, Gedisa, pp. 405-441

JARAÍZ, G. (2011): Intervención, barrio y Servicios Sociales Comunitarios, Madrid, Fundación Foessa.

KAEFER, F., ROPER, J. Y SINHA, P. (2015): “A Software-Assisted Qualitative Content Analysis of New Articles: Example and Reflections", Forum: Qualitative Social Research, 16 (2), pp.8-28.

KAUFMAN, L., \& ROUSSEEUW, P. J. (2005): Finding groups in data: an introduction to cluster analysis, EEUU, Wiley.

KOHLBACHER, F. (2006): “The Use os Qualitative Content Analysis in Case Study Reseacrh" Forum: Qualitative Social Research, 7 (1), Art. 21 doi:http://dx.doi. org/10.17169/fqs-7.1.75.

KRIPPENDORFF, K. (2004): Content Analysis: An Introduction to Its Methodology, Thousand Oaks (CA), Sage Publications

KVALE, S. (1996): Interviews: An introduction to Qualitative Research Interviewing. London, Sage Publications.

LÉRAT, P. (1995): Las lenguas especializadas, Barcelona, Ariel.

LIMA, J. L. y MANINI, P. M. (2016): "Metodología para el Análisis Cualitativo de Contenido integrado con la técnica de Mapas Mentales utilizando los softwares Nvivo y FreeMind”, Informação \& Informação, 21(3), pp. 63-100.

LIMA BANDEIRA, M. Y RUBAII, N. M. (2016). "El valor del análisis de discurso en los estudios comparativos de políticas públicas. El caso de aseguramiento de calidad en la educación superior en Colombia y Ecuador”. Estudios Políticos, 49, pp. 13-34. http://dx.doi.org/7533/udea.espo.n49a01

LÓPEZ FERRERO, C. (2002): "Aproximación al análisis de los discursos profesionales”. Revista Signos 35 (51-52), pp. 195-215. http://dx.doi.org/10.4067/5071809342002005100013

MAINGUENEAU, D. (2014): Discours et analyse du discours, París, Armand Colin.

Mendieta, D. y Esparcia, J. (2018): "Aproximación metodológica al análisis de contenidos a partir del discurso de los actores. Un ensayo de investigación social de procesos de desarrollo local (Loja, Ecuador)". EMPIRIA. Revista de Metodología de Ciencias Sociales n 39 ., pp. 15-47, DOI: 10.5944/empiria

MOLINA, J. (2017): Tutorial para el análisis de textos con el software Iramuteq. Disponible en: https://www.researchgate.net/publication/315696508_Tutorial_para_el_ analisis de textos_con_el_software_IRAMUTEQ [consulta: 13-09-18]

MORALES, A (2016): "Estudio lexicométrico de las sentencias de recursos de inconstitucionalidad del Tribunal Constitucional (2008-2013)". 13ème Journées internationales d'Analyse statistique des Données Textuelles. 7-10 juin 2016 Nice (France)

MORRIS, B. Y BASTIN, R. (2004): Expert knowledge, Nueva York, Berghahm Books. 
MOSCOVICI, S. (1961): El psicoanálisis, su imagen y su público, Buenos Aires, Huemul.

PALMQUIST, M. (2010). Writing guide: Content analysis. Fort Collins, CO: Colorado State University

PALTRIDGE, B. (2012): Discourse analysis: an introduction, London, Bloomsbury Academic

PÊCHEUX, M. (1969): L`analyse automatique du discours, Paris, Dunod.

PERKINS, D.D. (1995): "Speaking Truth to Power: Empowerment Ideology as Social Intervention and policy", American Journal of Community Psycology, 23, pp. 765794.

PIÑUEL, J.L. (2002): “Epistemología, metodología y técnicas del análisis de contenido", Estudios de Sociolingüística 3(1), pp. 1-42

PURCELL, T.W. (1998): "Indigenous knowledge and applied anthropology: questions of definition and direction". Human Organization 57, pp. 258-272.

REINERT, M. (1983): "Une méthode de classification descendante hiérarchique:application à l'analyse lexicalepar contexte". Les cahiers de l'analyse des données, 8(2), 187-198.

REINERT, M. (1987): “Classification Descendante Hierarchique et Analvse Lexicale par Contexte-Application au Corpus des Poesies D'A. Rihbaud". Bulletin of Sociological Methodology/Bulletin de Méthodologie Sociologique, 13(1), 53-90.

REINERT, M. (1998): "Quel objet pour une analyse statistique du discours? Quelques réflexions à propos de la réponse Alceste". JADT (pp. 557-569). Disponible en: http://w3dev.ualg.pt/ lfaisca/SMAD03/JADT_Reinert_1998.pdf [consulta: 01-1018]

REINERT, M. (2003). "Le rôle de la répétition dans la représentation du sens et son approche statistique par la méthode« ALCESTE»". SEMIOTICA-LA HAYE THEN BERLIN-, 147(1/4), 389-420

ROSA, M. Y ENCINA, J. (2004): "Democracias participativas desde las praxis locales" en Democracias participativas e intervención social comunitaria desde Andalucía, Sevilla, ACSUR, Atrapasueños y Universidad Pablo de Olavide

RUBIN, H.J. Y RUBIN. I. (1995): Qualitative interviewing: the art of hearing data, London, Sage Publications.

RUIZ BUENO, A, (2017): Trabajar con IRAMUTEQ: Pautas. Disponible en: http://diposit.ub.edu/dspace/bitstream/2445/113063/1/Trabajar_con_IRAMUTEQ_PAUTAS.pdf [consulta: 118-09-18]

RUIZ, E. Y GONZÁLEZ A. (2006): “Cómo conocen los técnicos? El conocimiento experto en la intervención participativa" en Sociología del Trabajo 57, pp.131-152.

SAARINEN, T. (2005). "“Quality" in the Bologna Process: From "Competitive Edge" to Quality Assurance Techniques", European Journal of Education, 40 (2), pp. 189204.

SALEM, A.; CABRÉ CASTELLVÍ, M. T.; ROMEU, L. (1990) Vocabulari de la lexicometria (espanyol-català-francès), Barcelona, Centre de Lexicometria. Divisió de Ciències Humanes i Socials.

SEGOVIA, P., BASULTO, O. Y ZAMBRANO, P. (2018): "Imaginarios sociales y representaciones: su aplicación a análisis discursivos en tres ámbitos diferentes". EMPIRIA. Revista de Metodología de Ciencias Sociales $n^{\circ} 41 .$, pp. 79-102 DOI/ empiria.41.2018.22605 
STAMANN, C., JANSSEN, M. y SCHREIER, M. (2016): "Searching for the Core: Defining Qualitative Content Analysis", Forum: Qualitative Social Research, 17(3), Art. 16, 24 p.

TROSBORG, A. (Ed.) (2000). Analysing Professional Genres, Amsterdam/Philadelphia, John Benjamins Publishing Company.

ZITTOUN, P. (2009). "Understanding Policy Change as a Discursive Problem", Journal of Comparative Policy Analysis, 11 (1), pp. 65-82.

ANEXO 1: Guion de estructura de las EP y GF. 


\begin{tabular}{|c|c|c|c|c|c|c|c|c|c|c|c|c|}
\hline 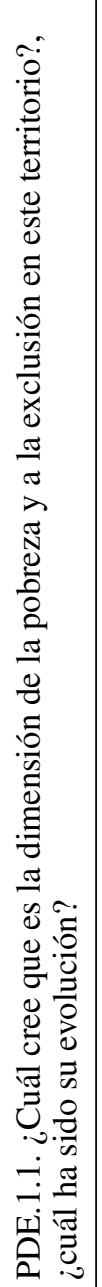 & 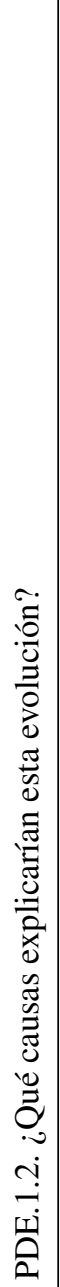 & 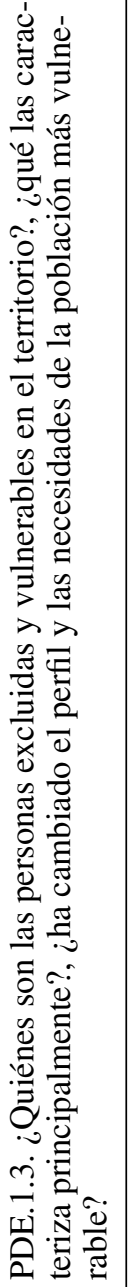 & 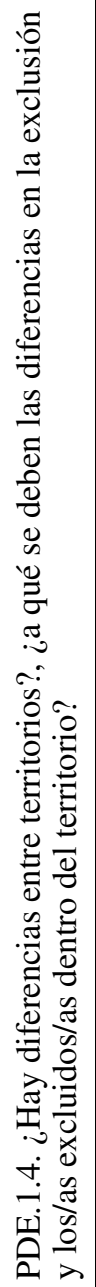 & 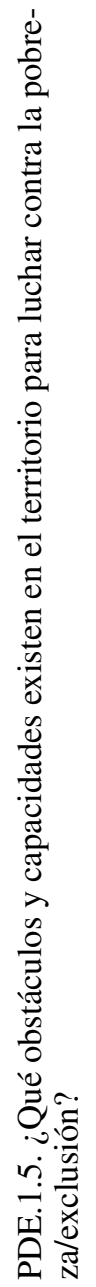 & 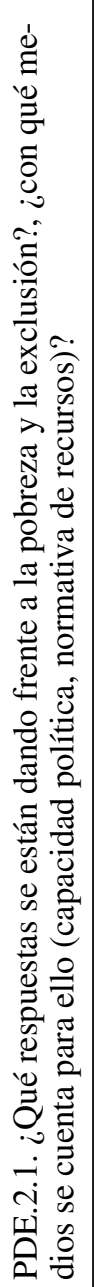 & 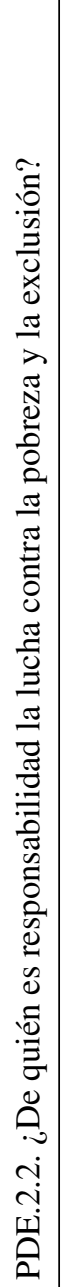 & 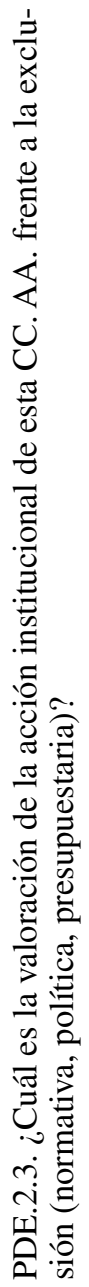 & 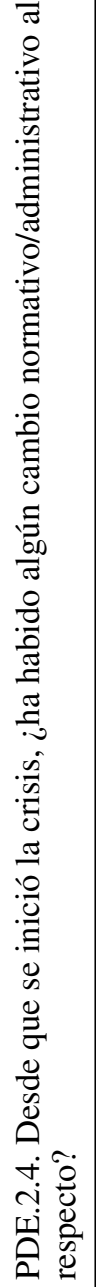 & 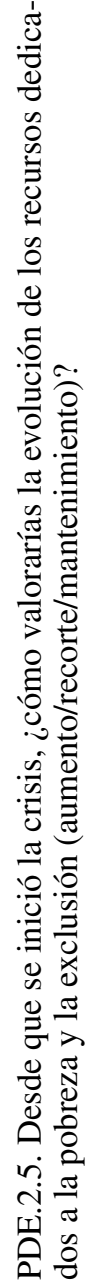 & 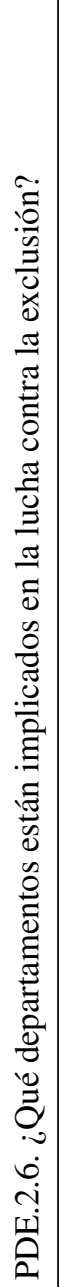 & 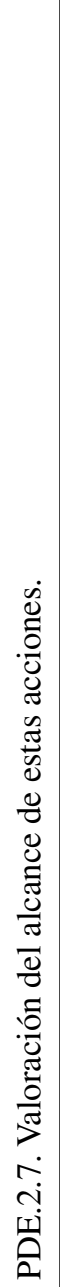 & 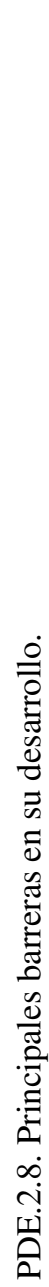 \\
\hline & & 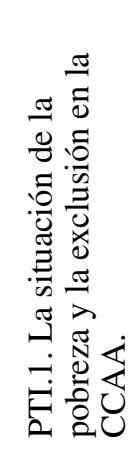 & & & & & & 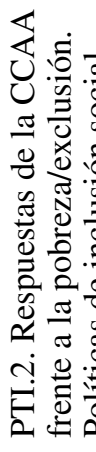 & & & & \\
\hline
\end{tabular}




\begin{tabular}{|c|c|c|c|c|c|c|c|c|c|c|c|c|}
\hline 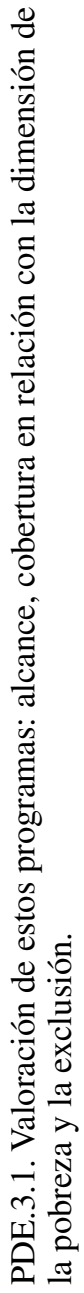 & 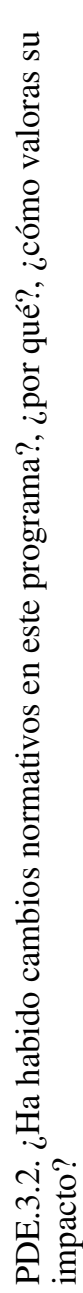 & 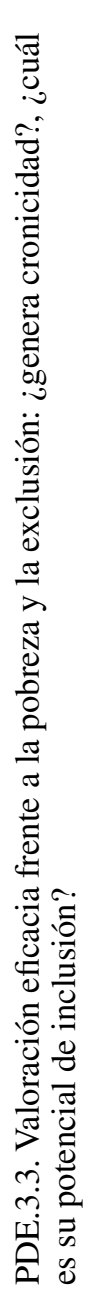 & 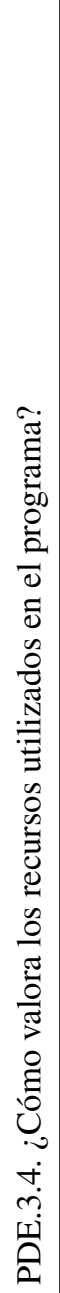 & 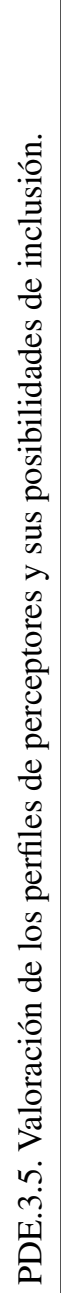 & 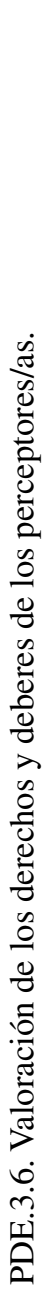 & 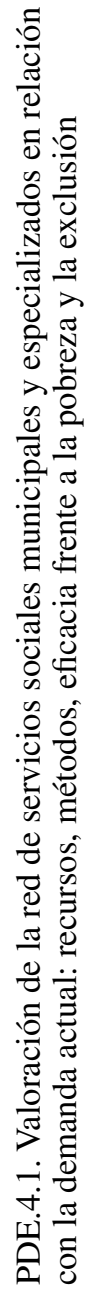 & 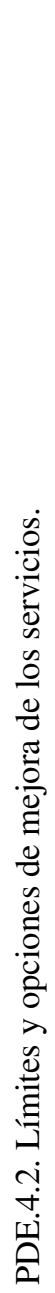 & 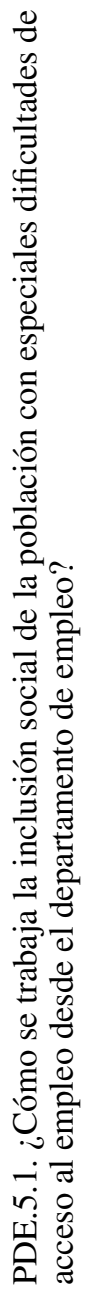 & 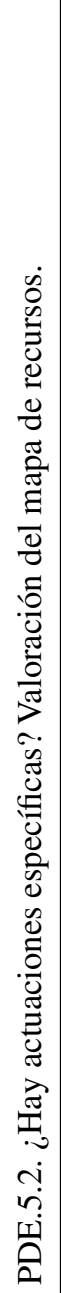 & 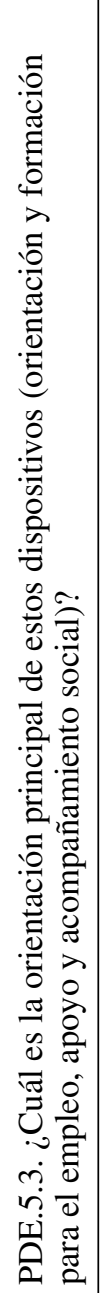 & 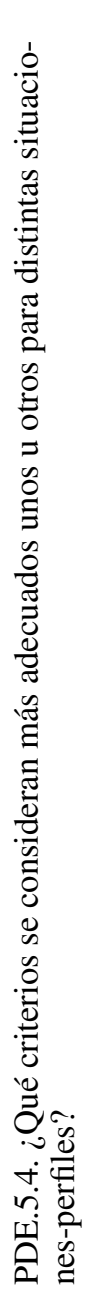 & 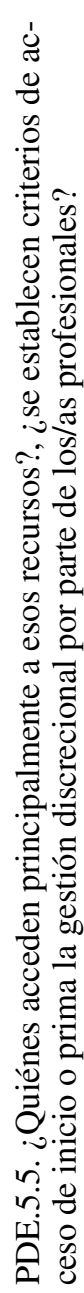 \\
\hline & & 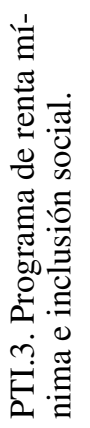 & & & & \multicolumn{2}{|c|}{ 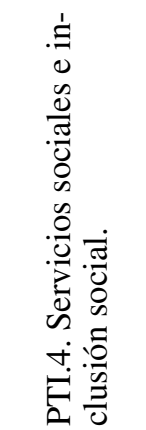 } & \multicolumn{5}{|c|}{ 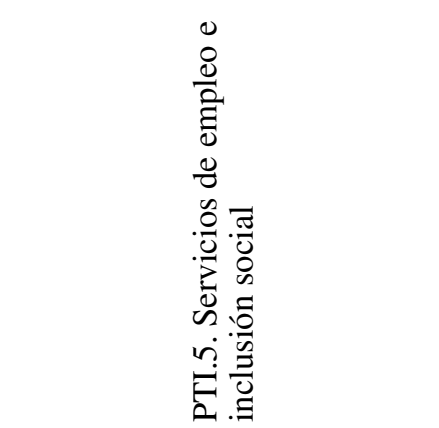 } \\
\hline
\end{tabular}




\begin{tabular}{|c|c|c|c|c|c|}
\hline 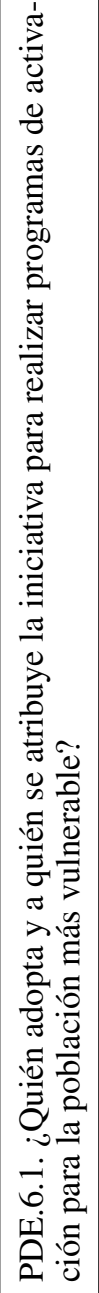 & 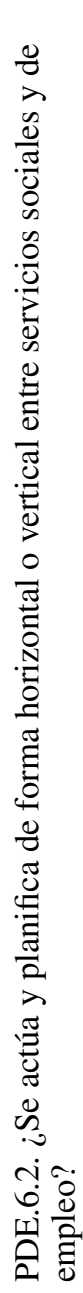 & 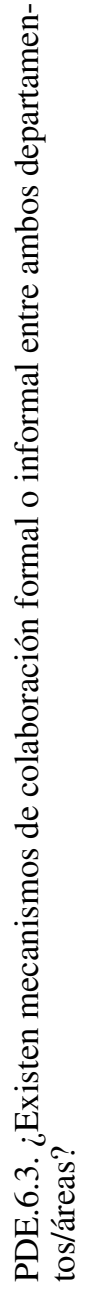 & 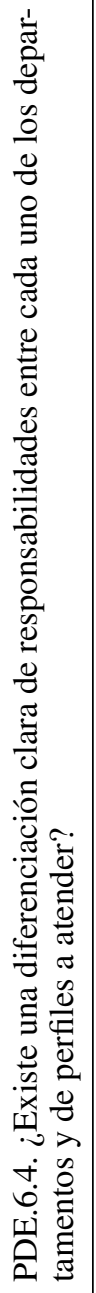 & 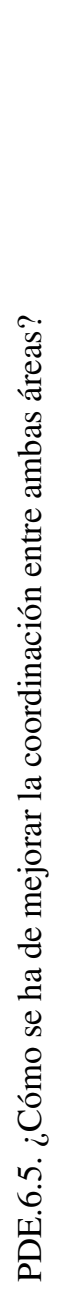 & 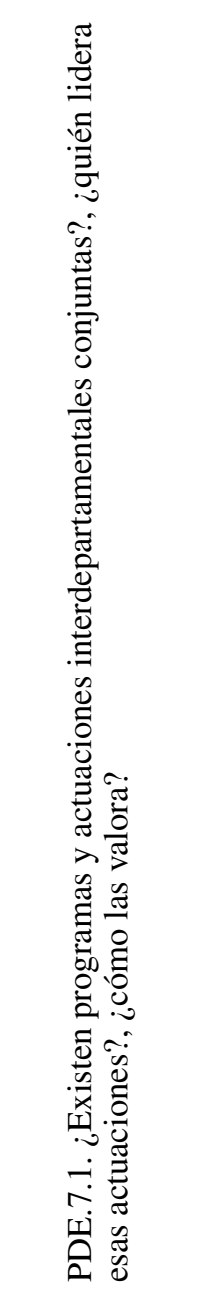 \\
\hline \multicolumn{5}{|c|}{ 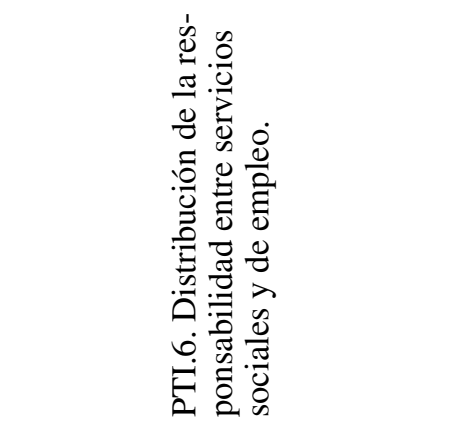 } & 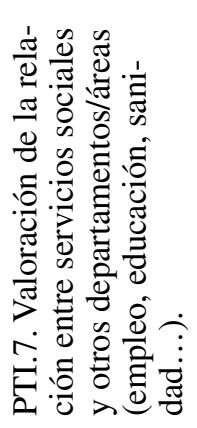 \\
\hline
\end{tabular}

EMPIRIA. Revista de Metodología de Ciencias Sociales. N. ${ }^{\circ} 45$ enero-abril, 2020, pp. 75-111. ISSN: 1139-5737, DOI/ empiria.43.2020.26305 


\begin{tabular}{|c|c|c|c|c|c|c|c|c|c|c|c|c|}
\hline 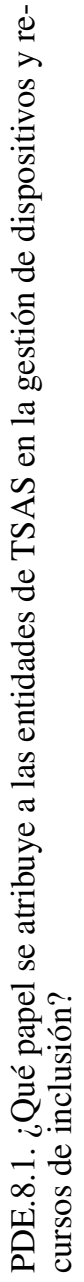 & 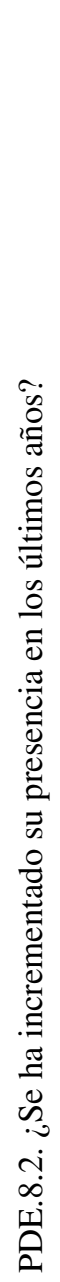 & 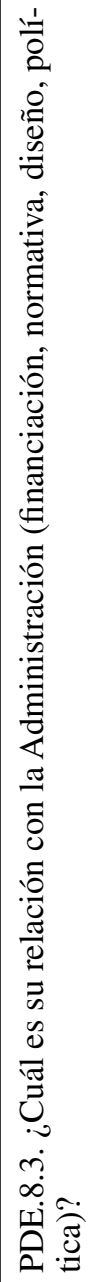 & 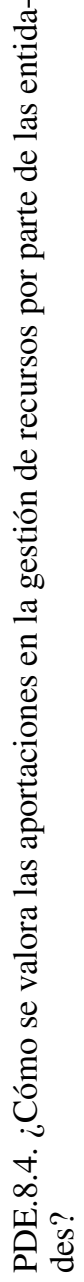 & 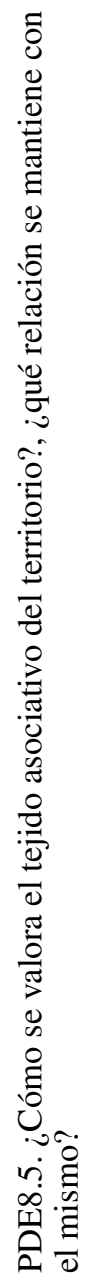 & 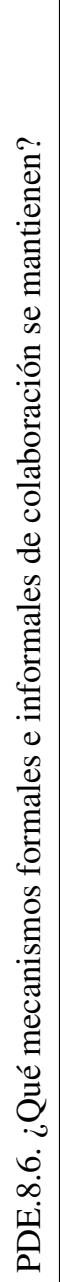 & 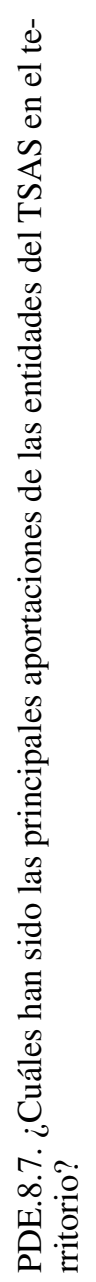 & 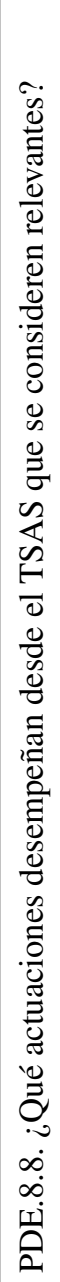 & 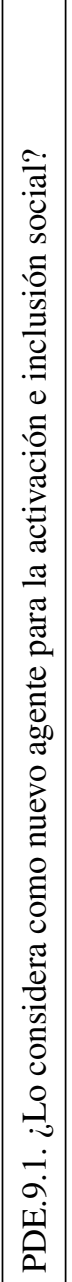 & 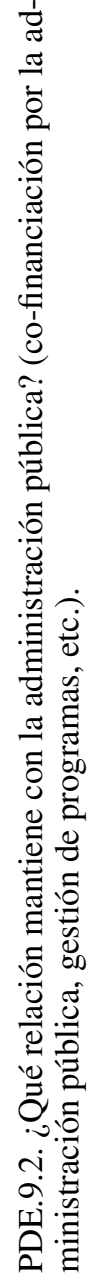 & 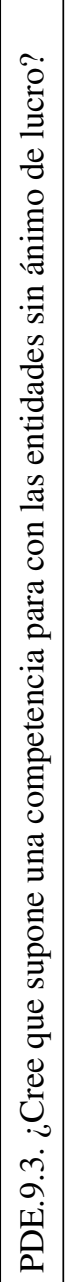 & 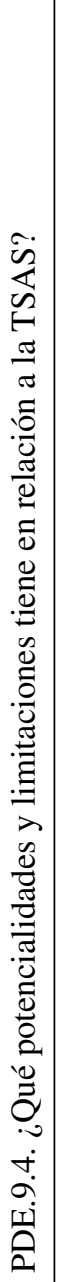 & 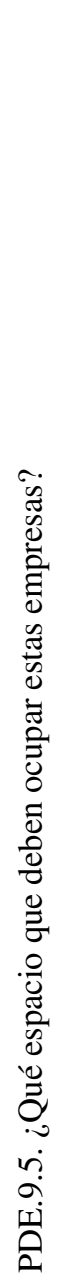 \\
\hline \multicolumn{8}{|c|}{ 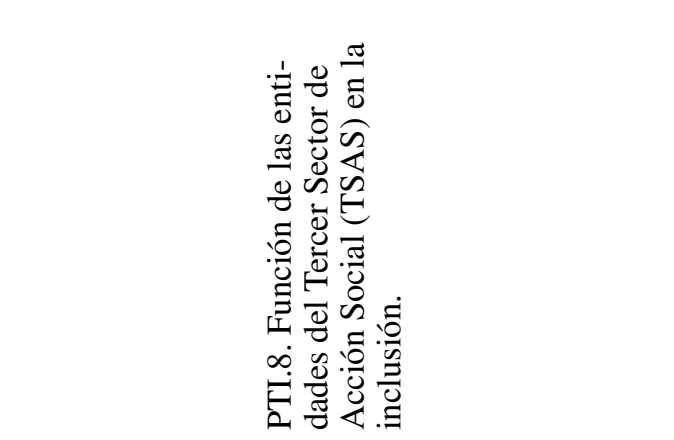 } & \multicolumn{5}{|c|}{ 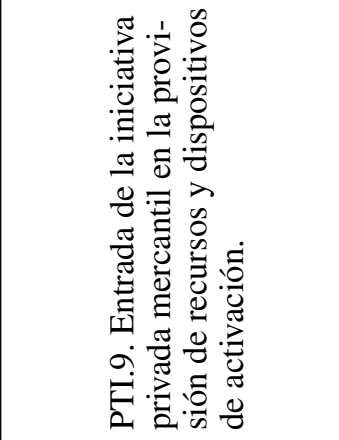 } \\
\hline
\end{tabular}




\begin{tabular}{|c|c|c|c|c|c|c|c|c|c|}
\hline 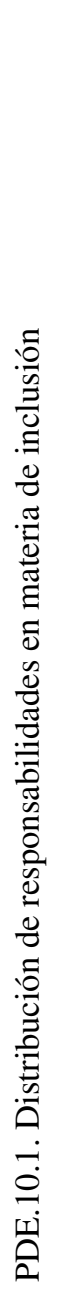 & 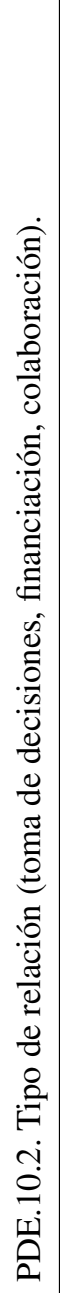 & 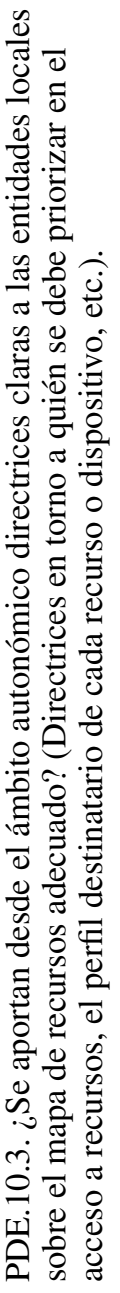 & 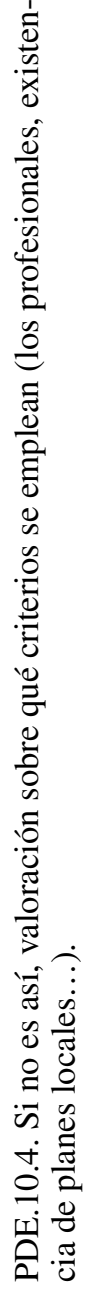 & 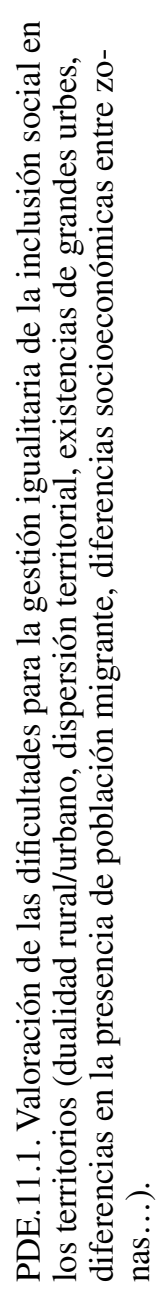 & 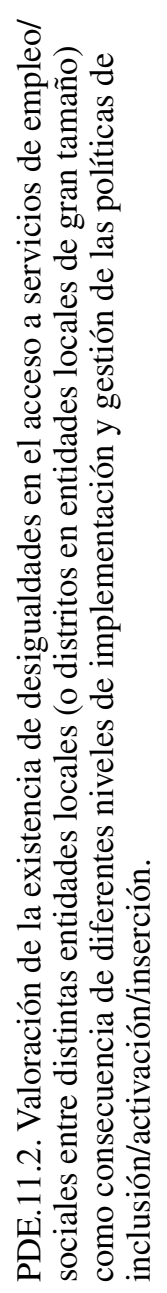 & 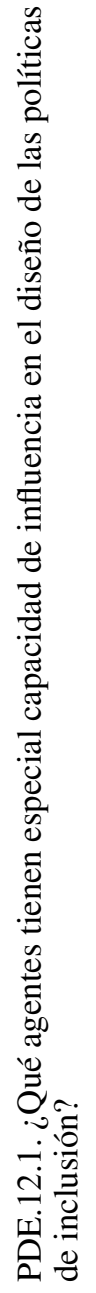 & 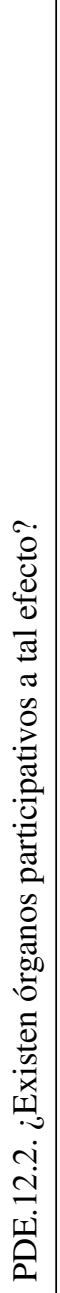 & 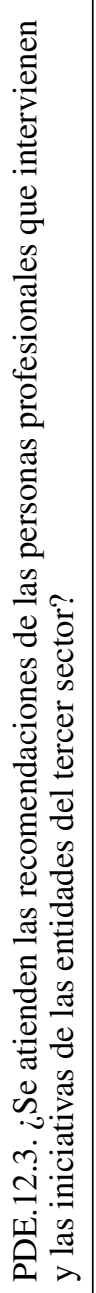 & 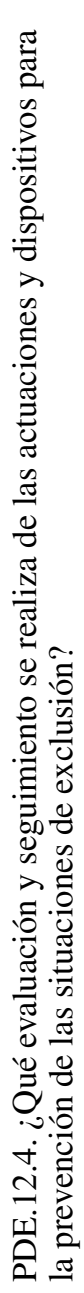 \\
\hline \multicolumn{4}{|c|}{ 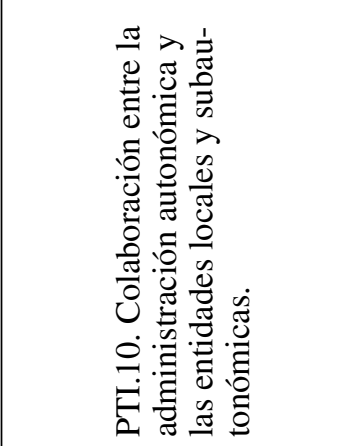 } & \multicolumn{2}{|c|}{ 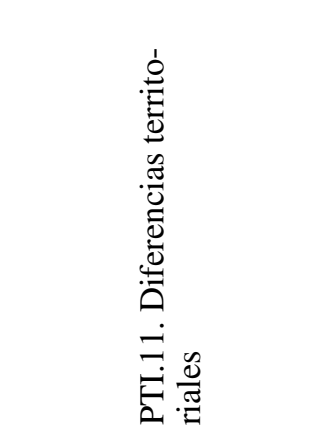 } & \multicolumn{4}{|c|}{ 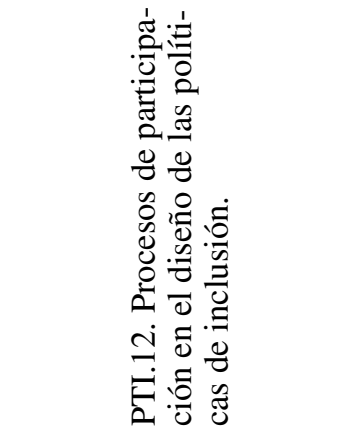 } \\
\hline
\end{tabular}




\begin{tabular}{|c|c|c|c|c|c|c|}
\hline 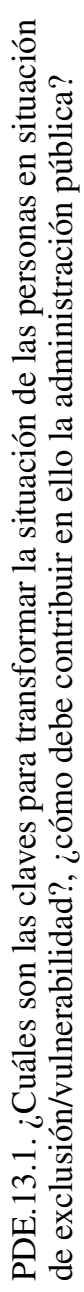 & 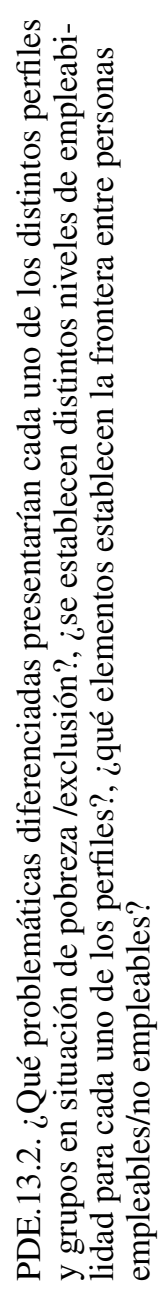 & 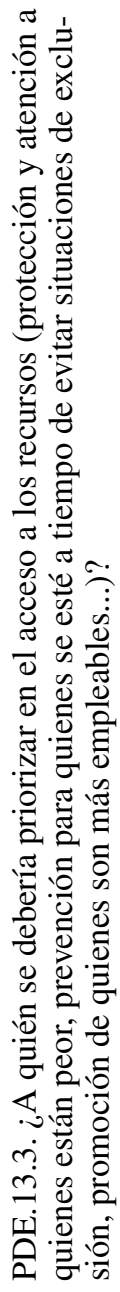 & 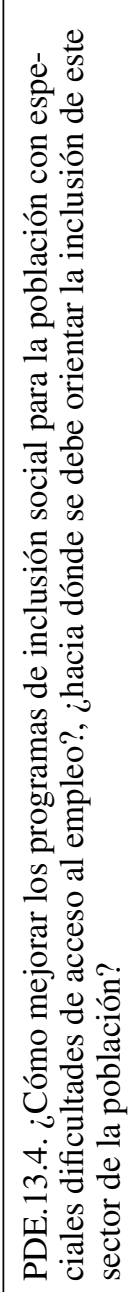 & 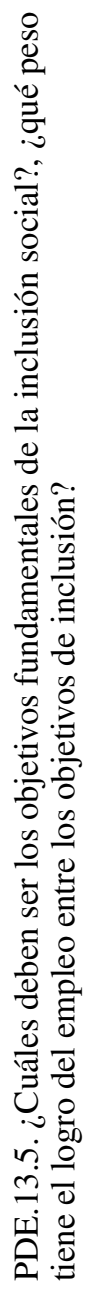 & 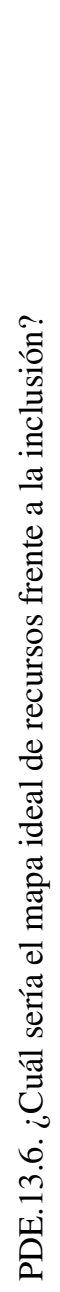 & 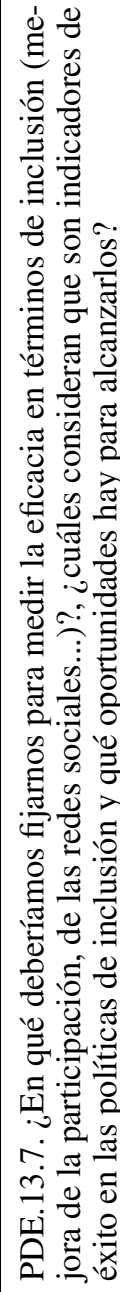 \\
\hline \multicolumn{7}{|c|}{ 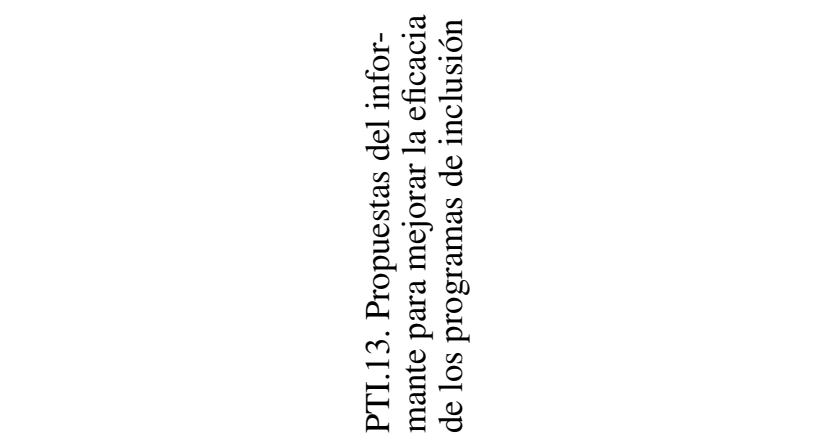 } \\
\hline
\end{tabular}


